International Journal of Modern Physics E, (C) World Scientific Publishing Company

\title{
NUCLEON STRUCTURE AND PARITY-VIOLATING ELECTRON SCATTERING
}

\author{
DOUGLAS H. BECK \\ Department of Physics, University of Illinois at Urbana-Champaign, 1110 West Green Street \\ Urbana, Illinois 61801-3080, USA \\ and \\ BARRY R. HOLSTEIN \\ Department of Physics-LGRT, University of Massachusetts \\ Amherst, Massachusetts 01003-4525, USA \\ Received (received date) \\ Revised (revised date)
}

\begin{abstract}
We review the area of strange quark contributions to nucleon structure. In particular, we focus on current models of strange quark vector currents in the nucleon and the associated parity-violating elastic electron scattering experiments from which vector and axial-vector currents are extracted.
\end{abstract}

\section{Introduction}

A description of the structure of hadrons in terms of quarks and gluons is one of the challenging problems in modern physics. Whereas QCD is taken to be the correct microscopic basis for such a description, efforts to solve the theory directly have thus far been unsuccessful for all but the shortest distance scale regimes. The short distance scale physics can indeed be calculated from QCD and matches very well with the body of deep-inelastic scatteringl and $\mathrm{e}^{+} \mathrm{e}^{-}$annihilation $\mathrm{d}$ data collected over the past 30 years. In the infinite momentum frame (where Bjorken scaling is manifest), the nucleon, for example, is viewed as primarily composed of its three valence quarks accompanied by ever increasing numbers of sea quarks and (especially) gluons as one looks to softer and softer parts of the wave function. The gluons, in fact, carry roughly half the nucleon momentum overall. In the same frame, the spin carried by quark degrees of freedom appears to be only partially responsible for the overall spin of the nucleon with the remainder being made up from quark orbital angular momentum and gluon spin.3

At the longest distance scales (on the order of the size of the hadron), the picture is at best incomplete. Models employing effective degrees of freedom, and which are more or less directly motivated by observation or by $\mathrm{QCD}$, are the present standards for this regime. Lattice QCD holds the promise of being able to calculate many of these low energy observables in the near future. It seems inevitable that 
some synthesis of lattice results and modeling will be required to lead us to the most economical approximate descriptions of hadron structure using the appropriate effective degrees of freedom - a path well worn in other areas of many-body physics. Careful measurements of low energy properties will be necessary to inform the development of these descriptions.

As is the case at the highest energies, the electroweak probe provides a well understood means of measuring observables at low energies as well. Measured at low energies, the electromagnetic properties of the nucleon provided the first indications that it is a composite particle, starting with the measurement of the proton magnetic moment by Stern et al. 1933 and continuing with the classic measurements by Hofstadter, et al. 0 of the proton charge and magnetic form factors in the 1950's. New measurements using both neutrino scattering scattering $\mathrm{l}$ provide complementary information to extend the understanding gained from the earlier studies.

With the unification of descriptions of the electromagnetic and weak interactions in the early 1970's, it became possible to consider comparing the electromagnetic and weak observables to extract more detailed information 10 In particular, because the photon and weak gauge bosons have precisely related couplings to the point-like quarks of the QCD lagrangian, it is possible to extract structure information according to quark flavor, as will be discussed in Section 2. Such determinations are particularly appropriate for comparison with lattice calculations because they represent partial, but potentially important, contributions to the overall low energy structure, providing a more detailed check than do global observables.

The contribution of strange quarks to the nucleon structure is of particular interest for developing our understanding, because it is exclusively part of the quarkantiquark sea. Further, these pairs reflect in part the gluon contributions that undoubtedly play an important role, as they do at smaller distance scales. The light (up and down) quark contributions to the sea may well have important differences relative to those of the strange quarks - a question that has not yet been thoroughly addressed. Nevertheless, there is at present no other technique that is directly sensitive to the quark sea at large distance scales.

That strange quarks make some contribution to nucleon structure is not in doubt. Measurement of charm production in deep-inelastic neutrino scattering provides both the $s$ and $\bar{s}$ momentum distributions. Recently an anaysis by the $\mathrm{NuTeV}$ collaboration has yielded the distinctly nonzero value 11

$$
\frac{2 \int_{0}^{1} d x(s+\bar{s})}{\int_{0}^{1} d x(u+\bar{u}+d+\bar{d})}=0.42 \pm 0.07 \pm 0.06
$$

for $Q^{2}=16 \mathrm{GeV}^{2}$ We note that the light sea quarks carry about $5 \%$ of the proton momentum in the parton model. Theoretical work involving light front methods has also suggested a significant strangeness content in the asymptotic region. 12 In

\footnotetext{
$\bar{a}$ There is at present no experimentally discernable difference in the $s$ and $\bar{s}$ distributions from these studies, but the analysis of the new round of experiments is continuing.
} 
this regard, the direct connection between light front and low energy manifestations of strangeness is difficult to establish, and more work is also needed here.

While it is not clear how to connect these deep-inelastic results to possible contributions at larger distance scales, the sum rules of spin-dependent deep-inelastic scattering do yield ground state properties. Defining the quark helicity content $\Delta q$ via

$$
\Delta q \sigma_{\mu}=<p, \sigma\left|\bar{q} \gamma_{\mu} \gamma_{5} q\right| p, \sigma>
$$

one has the constraint from $\vec{\ell} \vec{N}$ scattering (at first order in $\alpha_{s}$ )

$$
\int_{0}^{1} d x g_{1}^{p}(x)=\frac{1}{2}\left[\frac{4}{9} \Delta u+\frac{1}{9} \Delta d+\frac{1}{9} \Delta s\right]\left(1-\frac{\alpha_{s}\left(q^{2}\right)}{\pi}\right)
$$

When combined with the Bjorken sum rule and its $\mathrm{SU}(3)$ generalization

$$
\begin{aligned}
\Delta u-\Delta d & =g_{A}(0)=F+D \\
\Delta u+\Delta d-2 \Delta s & =3 F-D
\end{aligned}
$$

one finds the solution $\Delta u=0.81, \Delta d=-0.42, \Delta s=-0.11$, indicating a small negative value for the strange matrix element. This is the simplest analysis possible, however, and there are ambiguities associated both with the assumption about $S U(3)_{f}$ symmetry and with gluon contributions that are as yet unresolved.

There exists an alternative probe for this matrix element: neutral current elastic neutrino scattering. The point here is that the form of the Standard Model axial current is

$$
<N\left|A_{\mu}^{Z}\right| N>=\frac{1}{2}<N\left|\bar{u} \gamma_{\mu} \gamma_{5} u-\bar{d} \gamma_{\mu} \gamma_{5} d-\bar{s} \gamma_{\mu} \gamma_{5} s\right| N>
$$

which is purely isovector in the case that the strange matrix element vanishes and can therefore be exactly predicted from the known charged current axial matrix element. This experiment was performed at BNL and yielded a result 6

$$
\Delta s=-0.15 \pm 0.09
$$

consistent with that found from the deep inelastic sector, but a more precise value is needed 0

One of the original motivations for work in this area of strange quark contributions to nucleon structure was the analysis of the pion-nucleon sigma term 13 which also yields ground state (scalar) matrix elements. The basic idea behind the sigma term analysis is that one expects in the limit of vanishing quark masses that the nucleon mass should approach some nonzero value $M_{0}$ associated with the gluon content and the $\bar{q} q$ condensate. On the other hand, in the real world, with nonzero quark mass, the nucleon mass is modified to become

$$
M_{N}=M_{0}+\sigma_{s}+\sigma
$$


where, defining $\hat{m}=\left(m_{u}+m_{d}\right) / 2$,

$$
\sigma_{s}=\frac{1}{2 M_{N}}<N\left|m_{s} \bar{s} s\right| N>, \quad \sigma=\frac{1}{2 M_{N}}<N|\hat{m}(\bar{u} u+\bar{d} d)| N>
$$

are the contributions to the nucleon mass from explicit chiral symmetry breaking effects (i.e. non-zero quark mass) involving strange, and non-strange quarks respectively. One constraint in this regard comes from study of the hyperon masses, which yields

$$
\begin{aligned}
\delta & =\frac{\hat{m}}{2 M_{N}}<N|\bar{u} u+\bar{d} d-2 \bar{s} s| N> \\
& =\frac{3}{2} \frac{m_{\pi}^{2}}{m_{K}^{2}-m_{\pi}^{2}}\left(M_{\Xi}-M_{\Lambda}\right) \simeq 25 \mathrm{MeV}
\end{aligned}
$$

and increases to about $35 \mathrm{MeV}$ when higher order chiral corrections are included 14 A second constraint comes from analysis of $\pi N$ scattering, which says that $\sigma$ can be extracted directly if an isospin-even combination of amplitudes could be extrapolated via dispersion relations to the (unphysical) Cheng-Dashen point

$$
F_{\pi}^{2} D^{(+)}\left(s=M_{N}^{2}, t=m_{\pi}^{2}\right)=\sigma
$$

When this is done the result comes out to be $\sim 60 \mathrm{MeV}$, which is lowered to about 45 $\mathrm{MeV}$ by higher order chiral corrections 15 . If $\langle N|\bar{s} s| N\rangle=0$, as might be expected from a naive valence quark picture, then we would expect the value coming from the hyperon mass limit and that extracted from $\pi N$ scattering to agree. The fact that they do not can be explained by postulating the existence of a moderate strange quark matrix element

$$
f=\frac{<N|\bar{s} s| N>}{<N|\bar{u} u+\bar{d} d+\bar{s} s| N>} \simeq 0.1
$$

implying $M_{0} \simeq 765 \mathrm{Mev}$ and $\sigma_{s} \simeq 130 \mathrm{MeV}$ which seem quite reasonable. However, recent analyses by Olsson 10 and by Pavan 17 have suggested rather larger values $\sim 70-80 \mathrm{MeV}$ - for the sigma term, leading to $f \simeq 0.2, M_{0} \simeq 500 \mathrm{MeV}$ and $\sigma_{s} \simeq$ $375 \mathrm{MeV}$, which appear somewhat larger then one might intutively expect to find. Hence this problem as well represents work in progress. 18

In contrast to the axial and scalar matrix elements, the contribution of the $s$ quarks to the vector currents of the nucleon (ordinary charge and magnetization currents) can be determined more directly. The $s$ (and the $u$ and $d$ ) quark contributions are separated by means of comparison of neutral weak and electromagnetic elastic scattering measurements at low momentum transfers. 10.29 .26 The only assumptions in this case are that the proton and neutron obey charge symmetry 21 (essentially that under an isospin rotation $u$ quarks in the proton become $d$ quarks in the neutron and vice versa) and that the quarks are point-like, spin 1/2 Dirac particles. Therefore, such measurements provide a relatively clean basis from which to describe low energy hadron structure. 
As previously indicated, measurements of neutrino scattering and parity-violating electron scattering also determine axial-vector current matrix elements. In particular, whereas neutrino scattering involves only the axial current "seen" by the Z boson, in parity-violating electron scattering, the photon can couple to an effective axial current as first pointed out by Zel'dovich.22 In the case of the photon, the axial current coupling involves in addition exchange of a weak neutral boson, either between the electron and the target, or between quarks in the target itself. At present, the interpretation of this measure of the axial current is beginning to generate significant discussion.

In view of the high level of recent experimental as well as theoretical work in this area, it is a propitious time to provide an overview of this field, which is the purpose of the present article. The structure of our review is as follows. In Section 2 the observables in parity-violating electron scattering are enumerated and Section 3 describes the present status of, and relations among, the model calculations of these quantities. In Section 4 a brief summary is given of experimental techniques and the results to date are presented in Section 5. We conclude with a discussion of possible future directions.

\section{Observables}

\subsection{Form factors and quark currents}

The observables in electroweak electron-nucleon scattering, the overall electromagnetic and neutral weak currents (as indicated in Figure 1), may be related to the currents of the elementary quarks in the nucleon. Assuming the quarks are pointlike Dirac particles, the form of such currents is

$$
J_{\mu}^{i}=<N\left|\sum_{f=\text { flavors }} e_{f}^{i} \bar{q}_{f} \Gamma_{\mu} q_{f}\right| N>
$$

where $i$ denotes the electromagnetic or neutral weak currents and $\Gamma_{\mu}$ is $\gamma_{\mu}$ for the vector currents and $\gamma_{\mu} \gamma_{5}$ for the weak axial current. The electromagnetic charges of the quarks, the familiar $2 / 3(u, c, t)$ and $-1 / 3(d, s, b)$, are related to the neutral weak vector charges

$$
e_{f}^{Z}=2 T_{3, f}-4 e_{f}^{\gamma} \sin ^{2}\left(\theta_{W}\right)
$$

where $T_{3, f}$ is the weak isospin. 6 When the sum is taken over all quark flavors, the above form of the nucleon current is exact for point-like Dirac quarks.

Usually, the nucleon current is written as a function of the phenomenological elastic form factors (to account for the wave functions in Eq. 12). For example, the electromagnetic (vector) current is

$$
J_{\mu}^{\gamma}=\bar{N}\left(F_{1}\left(q^{2}\right) \gamma_{\mu}+i \frac{F_{2}\left(q^{2}\right)}{2 M} \sigma_{\mu \nu} q^{\nu}\right) N
$$

\footnotetext{
${ }^{b}$ The definition of the weak charge is the same as in Ref. B but, for examp a factor of 4 larger than Ref. 23; and a factor of 2 larger than that of the Particle Data Group 24
} 

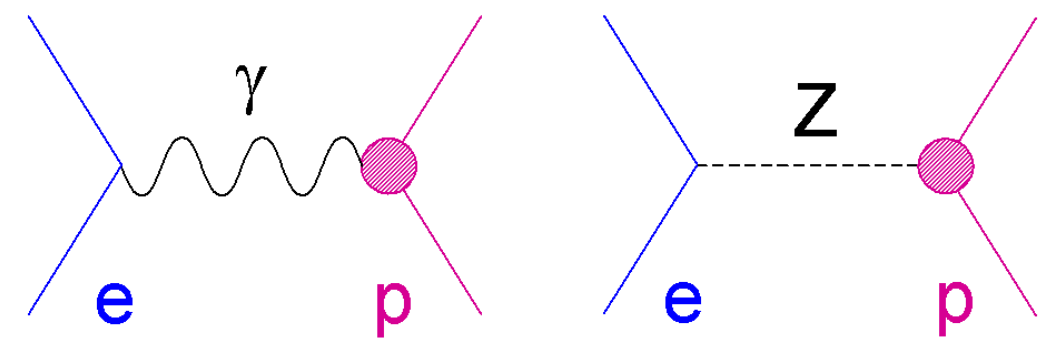

Fig. 1. Lowest order Feynman diagrams contributing to electron-nucleon scattering. The electroweak currents of the nucleon are indicated by the shaded disks.

where now the $N$ 's are nucleon spinors and the Dirac $\left(F_{1}\right)$ and Pauli $\left(F_{2}\right)$ form factors are normalized to unity and the nucleon anomalous magnetic moment, respectively, at $Q^{2}=0$. The more common form for $F_{1}$ and $F_{2}$ is in terms of the Sachs (charge and magnetic) form factors

$$
\begin{aligned}
G_{E} & =F_{1}-\frac{Q^{2}}{4 M^{2}} F_{2} \\
G_{M} & =F_{1}+F_{2}
\end{aligned}
$$

The axial current of the nucleon is similarly defined in terms of the form factor $G_{A}$

$$
J_{A, \mu}^{Z}=\bar{N} G_{A}\left(q^{2}\right) \gamma_{\mu} \gamma_{5} N .
$$

These form factors can also be expressed in terms of a linear combinations of the currents of the different flavors of quarks (as in Eq. 12). Factoring out the quark charges, the electromagnetic and neutral weak vector form factors can be written

$$
\begin{aligned}
G_{E, M}^{\gamma} & =\frac{2}{3} G_{E, M}^{u}-\frac{1}{3}\left(G_{E, M}^{d}+G_{E, M}^{s}\right) \\
G_{E, M}^{Z} & =\left(1-\frac{8}{3} \sin ^{2} \theta_{W}\right) G_{E, M}^{u}+\left(-1+\frac{4}{3} \sin ^{2} \theta_{W}\right)\left(G_{E, M}^{d}+G_{E, M}^{s}\right)
\end{aligned}
$$

Similarly, the neutral weak axial currents of the quarks can be identified in the overall axial current

$$
G_{A}=G_{A}^{u}-\left(G_{A}^{d}+G_{A}^{s}\right) .
$$

These equations illustrate the key point: the electromagnetic and neutral weak vector form factors (currents) represent different linear combinations of the same matrix elements of contributions from the different flavors of quarks.

It should be noted that antiquarks are implicitly included in the above definitions. Because they have charges of the opposite sign, quarks and antiquarks contribute to the matrix elements $G_{E, M, A}^{f}$ with opposite signs. For example, if the spatial distributions of $s$ and $\bar{s}$ quarks were the same, their charges would cancel everywhere and $G_{E}^{s}$ would vanish. 
Returning to Eqs. 17, it is straightforward to solve for the contributions of the three flavors in the case where one more observable can be found that depends on a different linear combination of these matrix elements. By assuming charge symmetry 21, the electromagnetic neutron form factors can be written in terms of the proton matrix elements. IIf we define the $G_{E}^{u}$, etc. above as contributions to the proton current, then assuming charge symmetry, the corresponding neutron form factors can be written as

$$
\begin{aligned}
G_{E, M}^{n, \gamma} & =\frac{2}{3} G_{E, M}^{d}-\frac{1}{3}\left(G_{E, M}^{u}+G_{E, M}^{s}\right) \\
G_{E, M}^{n, Z} & =\left(1-\frac{8}{3} \sin ^{2} \theta_{W}\right) G_{E, M}^{d}+\left(-1+\frac{4}{3} \sin ^{2} \theta_{W}\right)\left(G_{E, M}^{u}+G_{E, M}^{s}\right) \\
G_{A}^{n} & =G_{A}^{d}-\left(G_{A}^{u}+G_{A}^{s}\right)
\end{aligned}
$$

For the record, the vector current contributions of the three flavors may be written in terms of the three observables

$$
\begin{aligned}
& G_{E, M}^{u, p}=\left(3-4 \sin ^{2} \theta_{W}\right) G_{E,, M}^{p, \gamma}-G_{E, M}^{p, Z} \\
& G_{E, M}^{d, p}=\left(2-4 \sin ^{2} \theta_{W}\right) G_{E, M}^{p, \gamma}+G_{E, M}^{n, \gamma}-G_{E, M}^{p, Z} \\
& G_{E, M}^{s, p}=\left(1-4 \sin ^{2} \theta_{W}\right) G_{E,, M}^{p, \gamma}-G_{E, M}^{n, \gamma}-G_{E, M}^{p, Z}
\end{aligned}
$$

where again the contributions are shown explicitly to refer to the proton.

The actual matrix elements corresponding to the nucleon form factors are easy to write down in the Breit frame and in the low momentum transfer limit. The matrix elements corresponding to contributions of the different quark flavors are the corresponding projections of the overall matrix elements. These matrix elements are listed in Table 1 .

Table 1. Form factor matrix elements in the Breit frame 25 and in the low momentum transfer limit. The operator $J_{+}$is that of the normal spherical component of the current.

\begin{tabular}{ccc}
\hline form factor & Breit frame & low momentum transfer limit \\
\hline$G_{E}\left(Q^{2}\right)$ & $\frac{1}{2 M e}\left\langle N\left|J_{0}\right| N\right\rangle$ & $\chi_{f}^{\dagger} \chi_{i}\left(F_{1}-\frac{Q^{2}}{4 M^{2}} F_{2}\right)$ \\
$G_{M}\left(Q^{2}\right)$ & $-\frac{1}{2|\vec{q}| e}\left\langle N\left|J_{+}\right| N\right\rangle$ & $\chi_{f}^{\dagger} \vec{\sigma} \chi_{i} \times \vec{q} \frac{F_{1}+F_{2}}{2 M}$ \\
$G_{A}\left(Q^{2}\right)$ & $\langle N|\vec{A}| N\rangle$ & $\chi_{f}^{\dagger} \vec{\sigma} \chi_{i} G_{A}$ \\
\hline
\end{tabular}

Two quantities are often discussed in connection with the behavior of the strange quark contributions to these form factors at the lowest momentum transfers - the strangeness (charge) radius, $\rho^{s}$ and the magnetic moment contribution, $\kappa^{s}$. They

\footnotetext{
${ }^{c}$ The assumption is, as mentioned above, that in exchanging $u$ and $d$ quarks, $\bar{u}$ and $\bar{d}$ quarks, and vice versa, a proton becomes a neutron
} 
are defined by

$$
\begin{aligned}
\lim _{Q^{2} \rightarrow 0} G_{E}^{s}\left(Q^{2}\right) & =-\frac{1}{6} Q^{2}\left(\rho^{s}\right)^{2}, \text { and } \\
G_{M}^{s}\left(Q^{2}=0\right) & =\kappa^{s}
\end{aligned}
$$

A third quantity which is often calculated is a different version of the strangeness radius $-r_{s}^{2}$-measured via the Dirac form factor

$$
\lim _{Q^{2} \rightarrow 0} F_{1}^{s}\left(Q^{2}\right)=-\frac{1}{6} Q^{2} r_{s}^{2}
$$

\subsection{Higher order effects}

There are important contributions to electroweak electron-nucleon scattering beyond those appearing in Fig. 1. Examples of some of these processes are shown in Fig. 2. These can be considered radiative corrections to the ordinary weak $Z^{0}$
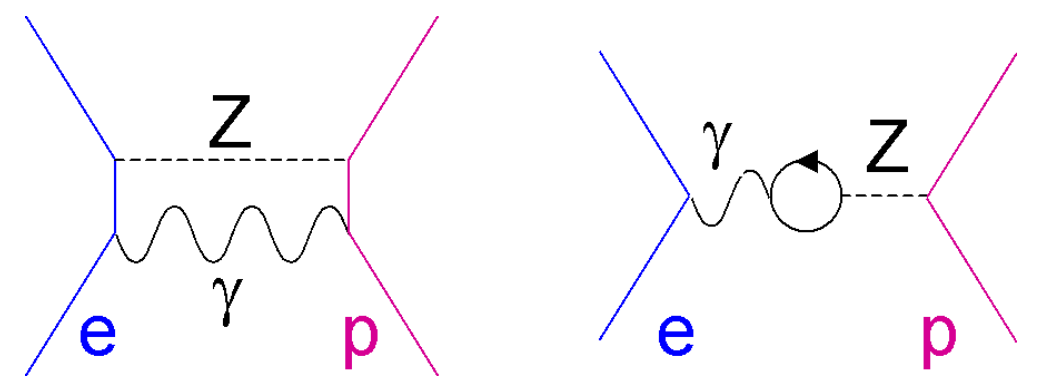

Fig. 2. Examples of higher order diagrams that contribute to electroweak electron-nucleon scattering.

exchange diagram and might be expected therefore to be $\mathcal{O}(1 \%)$ corrections to the lowest order predictions. This assumption is incorrect. The point is that in order to be parity-violating $Z^{0}$ exchange must involve either

$$
V\left(e^{-}\right) \times A(p) \quad \text { or } A\left(e^{-}\right) \times V(p)
$$

coupling. However, the weak vector coupling to the electron involves the factor $1-4 \sin ^{2} \theta_{W}$ and is strongly suppressed. Thus the primary sensitivity of a parityviolating electron scattering experiment is to the case where the weak axial coupling is to the electron and the weak hadronic interaction is vector in character. This can be seen explicitly below in Eqs. 29-34. However, this has the consequence that, since the piece involving the weak hadronic axial coupling is suppressed in leading order, the corresponding radiative corrections to this axial term can be $\mathcal{O}\left(\alpha /\left(1-4 \sin ^{2} \theta_{W}\right)\right.$ and quite significant. This expectation is borne out in detailed calculationsed, where corrections to the axial coupling term involving $G_{A}^{Z}$ are found to be as much as $30 \%$. However, this correction is also very uncertain for two reasons 
i) In diagram 2 a only the component of the box diagram with a nucleon intermediate state can be reliably computed. Contributions from pieces involving excited intermediate state hadrons are unknown.

ii) In diagram $2 \mathrm{~b}$ wherein $Z^{0}$ or $W^{ \pm}$exchange can occur purely within the hadronic system, leading to what is sometimes called the anapole moment, the calculations involve the full complexity of the hadronic state interacting with the quark loops. Although such effects have been estimated, uncertainties here can be easily $100 \% 26$.

Thus the axial current contribution to the asymmetry is not precisely calculable at the present time and a considerable uncertainly must be attributed to any such estimate. This uncertainty acts as an important systematic background which limits the precision to which strangeness effects can be measured, and this point will be emphasized below when the results of the SAMPLE experiment are discussed. On the other hand, the anapole related components have a physics interest in their own right, and are now beginning to be addressed.

\subsection{Parity-violating electron scattering}

The neutral weak current of the nucleon can be measured in either elastic neutrino scattering 0.27 or in parity-violating electron scattering. 20.29 .20 Ordinary, unpolarized electron scattering is, of course, dominated by the electromagnetic currents of the electron and nucleon. However, because the weak interaction violates parity (i.e. the weak current contains both vector and axial-vector contributions), the interference of the electromagnetic and weak currents violates parity. Observation of this small effect requires comparison of an experiment and its mirror image. In particular, the cross section contains a pseudoscalar component which will change signs in the mirror experiment. In parity-violating electron scattering, the mirror measurement is made by reversing the (pseudoscalar) beam helicity, $h=\hat{s} \cdot \hat{p}= \pm 1$, where $\hat{s}$ and $\hat{p}$ are the beam spin and momentum directions, respectively.

It is therefore the asymmetry

$$
A^{P V} \equiv \frac{\sigma_{+}-\sigma_{-}}{\sigma_{+}+\sigma_{-}}
$$

that is of interest since the cross section is

$$
\sigma \propto\left|\mathcal{M}^{\gamma}+\mathcal{M}^{Z}\right|^{2}
$$

The asymmetry comprises three terms, each reflecting the interference between the electromagnetic and neutral weak amplitudes (c.f. Fig. 1)

$$
A_{P V}=-\frac{G_{F} Q^{2}}{4 \pi \alpha \sqrt{2}} \frac{A_{E}+A_{M}+A_{A}}{D}
$$

where

$$
A_{E}=\epsilon(\theta) G_{E}^{\gamma} G_{E}^{Z}
$$




$$
\begin{aligned}
A_{M} & =\tau G_{M}^{\gamma} G_{M}^{Z}, \\
A_{A} & =-\left(1-4 \sin ^{2} \theta_{W}\right) \sqrt{\tau(1+\tau)\left(1-\epsilon(\theta)^{2}\right)} G_{M}^{\gamma} G_{A}^{Z}, \\
D & =\epsilon(\theta)\left(G_{E}^{\gamma}\right)^{2}+\tau\left(G_{M}^{\gamma}\right)^{2}, \text { and } \\
\epsilon(\theta) & =\left[1+2(1+\tau) \tan ^{2} \theta / 2\right]^{-1}
\end{aligned}
$$

where each of the three terms (electric, magnetic and axial) is a product of electromagnetic and weak form factors as expected. The overall scale of the asymmetry is set by the ratio of the neutral weak and electromagnetic propagators, i.e.

$$
\frac{\mathcal{M}^{Z}}{\mathcal{M}^{\gamma}} \sim \frac{Q^{2}}{\left(M^{Z}\right)^{2}}
$$

or about $10^{-4}$ at $Q^{2}=1 \mathrm{GeV}^{2}$. The differing angular dependence associated with these three terms can be used to separate the contributions experimentally. Whereas the magnetic term contributes independent of angle, the electric term vanishes at $180^{\circ}$ and the axial term vanishes at $0^{\circ}$.

It is these three terms in the asymmetry that are addressed by the experiments to be described in Sections 4 and 5. Before presenting these measurements, however, we compare and contrast the models used to describe the strange quark contributions to the nucleon vector currents.

\section{Theoretical Models}

As suggested above, a simple three quark picture of the nucleon yields zero for strange matrix elements. However, this is clearly too simplistic. Indeed, historically one of the first steps topwards a more realistic, physical decription was to consider the nucleon's strangeness content as a meson cloud effect - i.e. the feature that a ("dressed") nucleon can, for a short time consistent with the uncertainty principle, transform into a ("bare") nucleon plus multi-pion state - within which description one can intuitively, but only qualitatively, understand the origin of the nucleon anomalous moment and charge structure. In the case of the strangeness matrix elements, of course, it is not the pion cloud which is responsible for the effect but rather the transformation into states containing strange quarks - $K \Lambda, K \Sigma, \eta N$ - which yields a nonzero effect. Estimates based upon a simple one loop calculation establish the existence of the effect28, but, in view of its quantitative failures in predicting the nucleon's charge structure and magnetic moment, are certainly expected to be unreliable in giving anything other than the order of magnitude that one might expect.

A more contemporary way to describe the origin of nonzero strangeness current matrix elements is in terms of the quark sea, which can be represented in terms of the fragmentation of gluons into $q \bar{q}$ pairs. In this regard, the $s \bar{s}$ component is of particular interest since it represents the lightest pure sea degree of freedom in the nucleon. In fact from the quark sea viewpoint, an intriguing question becomes: since such sea effects are always present why does the simple valence quark model 
work so well in predicting low energy properties of the nucelon? Possible answers have been given to this question by Geiger and Isgur who point out that in the adiabatic approximation the effect of the virtual pairs is effectively to renormalize the string tension, which in the quark model is determined phenomenologically and therefore already includes such sea effects.29 A different answer is given by Kaplan and Manohar, who suggest that the primary effect of such pairs is to renormalize the current quarks of QCD into the constituent quarks used in phenomenological studies, so that again direct effects are hidden.10 Other issues which bear on this question are the origins of the OZI rule29 and the role of gluons in the dynamics of the strange quark sea. 30 In this paper we will not seek to address such trenchant questions, but rather to simply ask how the size of such strangeness current matrix elements can be understood from a theoretical perspective. As we shall see below, the calculation of quark sea effects within the hadronic medium is one of the more difficult problems in low energy physics and a definitive answer will not be possible. Consequently, we seek an approach which is model-independent and based on controlled approximations, but which is at the same time effective in establishing contact between theory and the experiment.

One such general principle is that of (broken) chiral symmetry, which is a property of the QCD Lagrangian which presumably underlies all interactions. A way in which one can attempt to insert the strictures of chiral invariance is through the use of chiral perturbation theory 31 , specifically through its heavy baryon version. 32 The basic idea is that in a world in which the light $(u, d, s)$ current quark masses vanish, the QCD Lagrangian日

$$
\mathcal{L}_{\mathrm{QCD}}=\bar{q}(i \not D-m) q-\frac{1}{2} \operatorname{tr} G_{\mu \nu} G^{\mu \nu} .
$$

would possess an exact $S U(3)_{L} \times S U(3)_{R}$ symmetry under independent left- and right-handed rotations of the light quarks

$$
q_{L} \rightarrow \exp \left(i \sum_{j} \lambda_{j} \alpha_{j}\right) q_{L}, \quad q_{R} \rightarrow \exp \left(i \sum_{j} \lambda_{j} \beta_{j}\right) q_{R}
$$

where here left and right refer to actions of the chirality projectors $P_{L, R}=\left(1 \pm \gamma_{5}\right) / 2$. The spontaneous symmetry breaking in the axial_sector which takes $S U(3) L \times$ $S U(3)_{R} \rightarrow S U(3)_{V}$ implies via Goldstone's theorem 33 the existence of eight massless pseudoscalar particles, which we identify with the $J^{P}=0^{-}$octet and an axial condensate, which we associate with the pion decay constant $F_{\pi}=92.4 \mathrm{MeV}$. (In the real world, of course, the current quark masses are small but nonzero, and so

\footnotetext{
${ }^{d}$ Here the covariant derivative is

$$
i D_{\mu}=i \partial_{\mu}-g A_{\mu}^{a} \frac{\lambda^{a}}{2},
$$
}

where $\lambda^{a}$ (with $a=1, \ldots, 8$ ) are the $\mathrm{SU}(3)$ Gell-Mann matrices, operating in color space, and the color-field tensor is defined by

$$
G_{\mu \nu}=\partial_{\mu} A_{\nu}-\partial_{\nu} A_{\mu}-g\left[A_{\mu}, A_{\nu}\right],
$$


these bosons are no longer massless but merely lighter than their hadronic siblings.) These bosons are described via the field

$$
U \equiv \exp \left(i \sum_{j} \lambda_{j} \phi_{j} / F_{\pi}\right)
$$

where $\lambda_{j}$ are the Gell-Mann matrices and $\phi_{j}$ are the pseudoscalar fields. The lowest order chiral effective Lagrangian is then

$$
\mathcal{L}_{2}=\frac{F_{\pi}^{2}}{4} \operatorname{Tr}\left(\partial_{\mu} U \partial^{\mu} U^{\dagger}\right)+\frac{B_{0}}{4} F_{\pi}^{2} \operatorname{Tr} m\left(U+U^{\dagger}\right) .
$$

where $m$ is the quark mass matrix and $B_{0}$ is a phenomenological constant related to the $\bar{q} q$ condensate. To order $\phi^{2}$ Eq. 41 reproduces the free meson Lagrangian and the Gell-Mann-Okubo relation 34

$$
3 m_{\eta}^{2}+m_{\pi}^{2}-4 m_{K}^{2}=0
$$

while at order $\phi^{4}$ it yields the Weinberg $\pi \pi$ scattering lengths 35

$$
a_{0}^{0}=\frac{7 m_{\pi}}{32 \pi F_{\pi}^{2}}, \quad a_{0}^{2}=-\frac{m_{\pi}}{16 \pi F_{\pi}^{2}}
$$

Such effective interaction calculations in the meson sector have been developed to a high degree over the past fifteen or so years, including the inclusion of loop contributions, in order to preserve crossing symmetry and unitarity. When such loop corrections are included one must augment the effective lagrangian to include "fourderivative" terms with arbitrary coefficients which must be fixed from experiment. This program, called chiral perturbation theory, has been enormously successful in describing low energy interactions in the meson sector.36 However, in order to discuss nucleons, we must extend it to consider baryons.

Writing down the lowest order such chiral Lagrangian at the $\mathrm{SU}(2)$ level is straightforward -

$$
\mathcal{L}_{\pi N}=\bar{N}\left(i \not D-m_{N}+\frac{g_{A}}{2} \psi \gamma \gamma_{5}\right) N
$$

where $g_{A}$ is the usual nucleon axial coupling in the chiral limit, the covariant derivative $D_{\mu}=\partial_{\mu}+\Gamma_{\mu}$ is given by

$$
\Gamma_{\mu}=\frac{1}{2}\left[u^{\dagger}, \partial_{\mu} u\right]-\frac{i}{2} u^{\dagger}\left(V_{\mu}+A_{\mu}\right) u-\frac{i}{2} u\left(V_{\mu}-A_{\mu}\right) u^{\dagger},
$$

and $u_{\mu}$ represents the axial structure

$$
u_{\mu}=i u^{\dagger} \nabla_{\mu} U u^{\dagger}
$$

The quantities $V_{\mu}, A_{\mu}$ represent external (non-dynamical) vector, axial-vector fields. Expanding to lowest order we find

$$
\begin{aligned}
\mathcal{L}_{\pi N} & =\bar{N}\left(i \not \partial-m_{N}\right) N+g_{A} \bar{N} \gamma^{\mu} \gamma_{5} \frac{1}{2} \vec{\tau} N \cdot\left(\frac{i}{F_{\pi}} \partial_{\mu} \vec{\pi}+2 \vec{A}_{\mu}\right) \\
& -\frac{1}{4 F_{\pi}^{2}} \bar{N} \gamma^{\mu} \vec{\tau} N \cdot \vec{\pi} \times \partial_{\mu} \vec{\pi}+\ldots
\end{aligned}
$$


which yields the Goldberger-Treiman relation, connecting strong and axial couplings of the nucleon system 37

$$
F_{\pi} g_{\pi N N}=m_{N} g_{A}
$$

Extension to SU(3) gives additional successful predictions - the baryon Gell-MannOkubo formula as well as the generalized Goldberger-Treiman relation. However, difficulties arise when one attempts to include higher order corrections to this formalism. The difference from the Goldstone case is that there now exist three dimensionful parameters $-m_{N}, m_{\pi}$ and $F_{\pi}$-in the problem rather than just $m_{\pi}$ and $F_{\pi}$. Thus loop effects can be of order $\left(m_{N} / 4 \pi F_{\pi}\right)^{2} \sim 1$ and we no longer have a reliable perturbative scheme. A consistent power counting mechanism can be constructed provided that we eliminate the nucleon mass from the leading order Lagrangian, as done in the procedure of Foldy and Wouthuysen 38 , which involves diagonalization of the particle and antiparticle components of the interaction. Equivalently one generates an expansion in powers of inverse nucleon mass by writing nucleon four-momenta as 39

$$
p_{\mu}=M v_{\mu}+k_{\mu}
$$

where $v_{\mu}$ is the four-velocity and satisfies $v^{2}=1$, while $k_{\mu}$ is a "small" off-shell momentum, with $v \cdot k<<M$. One can construct eigenstates of the projection operators $P_{ \pm}=\frac{1}{2}(1 \pm \psi)$, which in the rest frame select upper, lower components of the Dirac wavefunction, so that 40

$$
\psi=e^{-i M v \cdot x}\left(H_{v}+h_{v}\right)
$$

where

$$
H_{v}=P_{+} \psi, \quad h_{v}=P_{-} \psi
$$

The $\pi N$ Lagrangian can then be written in terms of $H, h$ as

$$
\mathcal{L}_{\pi N}=\bar{H}_{v} \mathcal{A} H_{v}+\bar{h}_{v} \mathcal{B} H_{v}+\bar{H}_{v} \gamma_{0} \mathcal{B}^{\dagger} \gamma_{0} h_{v}-\bar{h}_{v} \mathcal{C} h_{v}
$$

where the operators $\mathcal{A}, \mathcal{B}, \mathcal{C}$ have the low energy expansions

$$
\begin{aligned}
\mathcal{A} & =i v \cdot D+g_{A} u \cdot S+\ldots \\
\mathcal{B} & =i \not D^{\perp}-\frac{1}{2} g_{A} v \cdot u \gamma_{5}+\ldots \\
\mathcal{C} & =2 M+i v \cdot D+g_{A} u \cdot S+\ldots
\end{aligned}
$$

Here $D_{\mu}^{\perp}=\left(g_{\mu \nu}-v_{\mu} v_{\nu}\right) D^{\nu}$ is the transverse component of the covariant derivative and $S_{\mu}=\frac{i}{2} \gamma_{5} \sigma_{\mu \nu} v^{\nu}$ is the Pauli-Lubanski spin vector and satisfies

$$
S \cdot v=0, \quad S^{2}=-\frac{3}{4}, \quad\left\{S_{\mu}, S_{\nu}\right\}=\frac{1}{2}\left(v_{\mu} v_{\nu}-g_{\mu \nu}\right), \quad\left[S_{\mu}, S_{\nu}\right]=i \epsilon_{\mu \nu \alpha \beta} v^{\alpha} S^{\beta}
$$

We observe that the two components $\mathrm{H}, \mathrm{h}$ are coupled in Eq. 52. However, the system may be diagonalized by use of the field transformation

$$
h^{\prime}=h-\mathcal{C}^{-1} \mathcal{B} H
$$


in which case the Lagrangian becomes

$$
\mathcal{L}_{\pi N}=\bar{H}_{v}\left(\mathcal{A}+\left(\gamma_{0} \mathcal{B}^{\dagger} \gamma_{0}\right) \mathcal{C}^{-1} \mathcal{B}\right) H_{v}-\bar{h}_{v}^{\prime} \mathcal{C} h_{v}^{\prime}
$$

The piece of the Lagrangian involving $H$ contains the mass only in the operator $\mathcal{C}^{-1}$ and is the effective Lagrangian that we seek. The remaining piece involving $h_{v}^{\prime}$ can be thrown away, as it does not couple to the $H_{v}$ physics. (In path integral language we simply integrate out this component yielding an uninteresting overall constant.)

Of course, for applications involving strangeness, we must generalize the above to $\mathrm{SU}(3)$ and the lowest order heavy baryon chiral Lagrangian becomes

$$
\mathcal{L}=i \operatorname{tr}(\bar{B} v \cdot D B)+2 \operatorname{tr}\left(\bar{B} S^{\mu}\left[D\left\{A_{\mu}, B\right\}+F\left[A_{\mu}, B\right]\right]\right)
$$

where $D, F$ are the usual SU(3) axial couplings with $D=F=g_{A}$. When loops are included one can be concerned about the convergence of such a chiral series, but the above formalism provides at least a useful framework for our discussions. The inclusion of loops also requires the insertion of phenomenological counterterms in order to absorb divergences. In our case we shall represent the strangeness current in terms the electromagnetic and baryon number currents -

$$
J_{\mu}^{e m}(I=1)=\bar{q} \frac{\lambda_{3}}{2} \gamma_{\mu} q, \quad J_{\mu}^{e m}(I=0)=\bar{q} \frac{\lambda_{8}}{2 \sqrt{3}} \gamma_{\mu} q, \quad J_{\mu}^{B}=\frac{1}{3} \bar{q} \gamma_{\mu} q
$$

via

$$
\bar{s} \gamma_{\mu} s=J_{\mu}^{B}-2 J_{\mu}^{e m}(I=0)
$$

and the appropriate counterterms are given by 41

$$
\begin{aligned}
\Delta \mathcal{L}_{e m}^{I=1} & =\frac{e}{\Lambda_{\chi}} \epsilon_{\mu \nu \alpha \beta} v^{\alpha}\left\{b_{+} \operatorname{tr}\left(\bar{B} S^{\beta}\left\{\lambda_{3}, B\right\}\right)+b_{-} \operatorname{tr}\left(\bar{B} S^{\beta}\left[\lambda_{3}, B\right]\right)\right\} F^{\mu \nu} \\
& -\frac{e}{\Lambda_{\chi}^{2}}\left\{c_{+} \operatorname{tr} \bar{B}\left\{\lambda_{3}, B\right\}+c_{-} \operatorname{tr} \bar{B}\left[\lambda_{3}, B\right]\right\} v_{\mu} \partial_{\nu} F^{\mu \nu} \\
\Delta \mathcal{L}_{e m}^{I=0} & =\frac{e}{\sqrt{3} \Lambda_{\chi}} \epsilon_{\mu \nu \alpha \beta} v^{\alpha}\left\{b_{+} \operatorname{tr}\left(\bar{B} S^{\beta}\left\{\lambda_{8}, B\right\}\right)+b_{-} \operatorname{tr}\left(\bar{B} S^{\beta}\left[\lambda_{8}, B\right]\right)\right\} F^{\mu \nu} \\
& -\frac{e}{\sqrt{3} \Lambda_{\chi}^{2}}\left\{c_{+} \operatorname{tr} \bar{B}\left\{\lambda_{8}, B\right\}+c_{-} \operatorname{tr} \bar{B}\left[\lambda_{8}, B\right]\right\} v_{\mu} \partial_{\nu} F^{\mu \nu} \\
\Delta \mathcal{L}_{B} & =\frac{b_{0}}{\Lambda_{\chi}} \epsilon_{\mu \nu \alpha \beta} v^{\alpha} \operatorname{tr} \bar{B} S^{\beta} B Z^{\mu \nu}-\frac{c_{0}}{\Lambda_{\chi}^{2}} \operatorname{tr} \bar{B} B v_{\mu} \partial_{\nu} Z^{\mu \nu}
\end{aligned}
$$

Here $\Lambda_{\chi}=4 \pi F_{\pi} \sim 1 \mathrm{GeV}$ is the chiral scale parameter and is inserted in order that the parameters $b_{i}, c_{i}$ should be $\mathcal{O}(1)$ while $F^{\mu \nu}, Z^{\mu \nu}$ are field tensors for external photon and Z-boson fields respectively.

${ }^{e}$ Indeed, as we shall discuss below, simple SU(3) loop calculations in heavy baryon chiral perturbation theory generate nonanalytic terms which are large and phenomenologically problematic. 
One can then represent the experimental values of strangeness anomalous moments and charge radii (see Eqs. 25) in terms of the sum of counterterm and loop contributions as

$$
\begin{aligned}
\kappa^{a} & =\kappa_{\text {loop }}^{a}+\left(\frac{2 M_{B}}{\Lambda_{\chi}}\right) b^{a} \\
\rho^{a} & =\rho_{\text {loop }}^{a}-\left(\frac{2 M_{B}}{\Lambda_{\chi}}\right)^{2} c^{a}
\end{aligned}
$$

where the one-loop contributions to the strangeness form factors are 41

$$
\begin{aligned}
\kappa_{\text {loop }}^{s}= & 2 \pi \frac{M_{N} m_{K}}{\Lambda_{\chi}^{2}}\left[\frac{1}{6}(3 F+D)^{2}+\frac{3}{2}(D-F)^{2}\right] \\
\rho_{\text {loop }}^{s}= & \left(\frac{M_{N}}{\Lambda_{\chi}}\right)^{2}\left[\frac{1}{\epsilon}-\gamma-\ln \frac{m_{K}^{2}}{4 \pi \mu^{2}}\right] \\
& \left\{1+\frac{5}{3}\left[\frac{1}{6}(3 F+D)^{2}+\frac{3}{2}(D-F)^{2}\right]\right\}
\end{aligned}
$$

Here dimensional regularization has been used with $\epsilon=(4-d) / 2$. Similar forms obtain for the electromagnetic loop contributions and in this way, using experimental values for the neutron and proton form factors one can obtain values for the counterterms $c_{ \pm}, b_{ \pm}-b_{+} \simeq 1.4, b_{-} \simeq 0.9, c_{+} \simeq-1.9, c_{-} \simeq 0.9$. (Note that these values are $\mathcal{O}(1)$, as expected from chiral counting arguments.) The values which we need in order to evaluate the strangeness terms ard $f$

$$
\begin{aligned}
& b^{s}=b_{0}-2\left[b_{-}-\frac{1}{3} b_{+}\right] \\
& c^{s}=c_{0}-2\left[c_{-}-\frac{1}{3} c_{+}\right]
\end{aligned}
$$

so that if values for $c_{0}, b_{0}$ were known corresponding to those for $c_{ \pm}, b_{ \pm}$our problem would be solved. Unfortunately this is not the case. Indeed, chiral perturbation theory relies on experiment to determine those quantities whose value is not required by chiral invariance so that in our case we require experimental values of the strangeness anomalous moment and charge radius in order to determine the missing counterterms - the theory is nonpredictive. As discussed below, it is possible to get around this difficulty by assuming a model, such as vector dominance, but this is beyond the basic strictures required by chiral invariance alone. An interesting exception to this chiral non-predictability arises, however, if one considers the $q^{2}$-dependence of the strange magnetic form factor, as pointed out by Hemmert, Meissner and Steininger. 42 In this case, although the strange magnetic moment itself is not predicted, the momentum tranfer dependence of the magnetic form factor is determined by the kaon loop diagram so that one has the model-independent

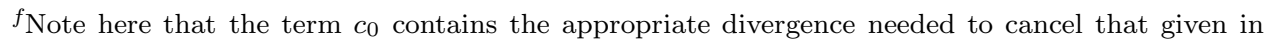
the loop calculation.
} 
prediction

$$
\begin{aligned}
F_{2}^{s}\left(q^{2}\right)-F_{2}^{s}(0)= & \frac{M_{N} m_{K}}{24 \pi F_{\pi}^{2}}\left(5 D^{2}-6 D F+9 F^{2}\right) f\left(q^{2}\right) \\
\text { where } & f\left(q^{2}\right)=-\frac{1}{2}+\frac{1+\frac{q^{2}}{4 m_{K}^{2}}}{2 \sqrt{\frac{q^{2}}{4 m_{K}^{2}}}} \tan ^{-1}\left(\sqrt{\frac{q^{2}}{4 m_{K}^{2}}}\right)
\end{aligned}
$$

One can use this expression in order to extrapolate from negative $q^{2}$, where experiments are done to $q^{2}=0$, which is where most theoretical calculations are performed. In the case of the SAMPLE experiment (see Section 5), this produces a shift of about 0.2 nucleon magnetons.

\subsection{Vector dominance}

Despite the inability of chiral methods to provide predictions for strangeness vector currents, the above formalism does provide a useful basis from which to take our calculation further. What we require are (of necessity model-dependent) methods by which to estimate the size of the chiral counterterms. In principle lattice methods can be used in this regard, and work in this area is in progress. 43 However, reliable estimation of subtle quark sea effects requires proper inclusion of quark loop contributions, while published calculations involve the quenched approximation, wherein quark-antiquark loop effects are neglected 44 Thus we require alternative means, which implies resorting to various hadronic models. An approach which has proved useful in the chiral mesonic sector is to estimate the size of counterterms via saturation by t-channel vector and axial-vector meson exchange contributions 45 In this way five of the ten $\mathcal{O}\left(p^{4}\right)$ Gasser-Leutwyler counterterms evaluated at a renormalization scale $\mu=m_{\rho}$ are reproduced rather successfully. In particular, the pion charge radius is predicted to be given predominantly in terms rho-meson exchange with only a small $(<10 \%)$ loop contribution. This, of course, is simply the vector dominance assumption, which is known to work well for this quantity. It then seems reasonable to attempt a similar calculation in the realm of baryons. For this purpose, it is useful to emply a tensor representation of the vector meson fields, as this automatically builds in proper asymptotic properties. 11 In particular, defining a VNN effective Lagrangian in terms of couplings $G_{T}, G_{V}$ via 41

$$
\mathcal{L}_{V N N}=2 G_{T} \epsilon_{\mu \nu \alpha \beta} v^{\alpha} \bar{B} S^{\beta} B V^{\mu \nu}+\frac{G_{V}}{\Lambda_{\chi}} \bar{B} B v_{\mu} D_{\nu} V_{\mu \nu}
$$

as well as a gauge invariant vector-meson photon coupling

$$
\mathcal{L}_{V \gamma}=\frac{e F_{V} \Lambda_{\chi}}{\sqrt{2}} F_{\mu \nu} V^{\mu \nu}
$$

one finds contributions to the charge and magnetic form factors

$$
F_{1}\left(q^{2}\right)=\sqrt{2} G_{V} F_{V} \frac{Q^{2}}{m_{V}^{2}-q^{2}}
$$




$$
F_{2}\left(q^{2}\right)=4 \sqrt{2} G_{T} F_{V} \frac{M_{N} \Lambda_{\chi}}{m_{V}^{2}} \frac{m_{V}^{2}}{m_{V}^{2}-q^{2}}
$$

One can then identify the counterterm predictions in terms of

$$
\begin{aligned}
b & =2 \sqrt{2} G_{T} F_{V}\left(\frac{\Lambda_{\chi}}{m_{V}}\right)^{2} \\
c & =\sqrt{2} G_{V} F_{V}\left(\frac{\Lambda_{\chi}}{m_{V}}\right)^{2}
\end{aligned}
$$

where we have for simplicity omitted the isospin designations. For the nucleons, the experimental electromagnetic form factors together with data on the leptonic decay of the vector mesons allows a determination of the couplings $G_{V}, G_{T}, F_{V}$. However, lacking knowledge of the couplings $F_{V}$ associated with the strange matrix elements $<0\left|\bar{s} \gamma_{\mu} s\right| V>$ we have no predictive power in the strange sector.

Nevertheless progress can be made by invoking the vector meson wavefunction quark content 46 The idea here is to write down a dispersion relation approach to these form factors and to assume vector meson dominance

$$
\begin{aligned}
& F_{1}^{a}\left(q^{2}\right)=F_{1}^{a}+\sum_{V} \frac{q^{2} a_{V}^{a}}{m_{V}^{2}-q^{2}} \\
& F_{2}^{a}\left(q^{2}\right)=\sum_{V} \frac{m_{V}^{2} b_{V}^{a}}{m_{V}^{2}-q^{2}}
\end{aligned}
$$

Here the isoscalar residues at the vector meson poles have been determined by Höhler et al. 47 and independently by Mergell et al 48 in terms of a fit involving $\omega, \phi$ and one higher mass vector meson $V^{\prime}$. Clearly at least two such poles are required in order to obtain the observed dipole dependence while a third pole was used in order to achieve a reasonable $\chi^{2}$ for the fit. Having such residues one can then evaluate the counterterm contributions in terms of

$$
\begin{aligned}
b^{a} & =\left(\frac{\Lambda_{\chi}}{2 M_{N}}\right) \sum_{V} b_{V}^{a} \\
c^{a} & =\sum_{V}\left(\frac{\Lambda_{\chi}}{m_{V}}\right)^{2} a_{V}^{a}
\end{aligned}
$$

Jaffe then pointed out that one could evaluate the strange residues at the $\omega, \phi$ poles in terms of the isoscalar electromagnetic residues vial

$$
\begin{aligned}
& \frac{a_{\omega}^{s}}{a_{\omega}^{I=0}}=-\frac{\sqrt{6} \sin \epsilon}{\sin \left(\epsilon+\theta_{0}\right)} \\
& \frac{a_{\phi}^{s}}{a_{\phi}^{I=0}}=-\frac{\sqrt{6} \cos \epsilon}{\cos \left(\epsilon+\theta_{0}\right)}
\end{aligned}
$$

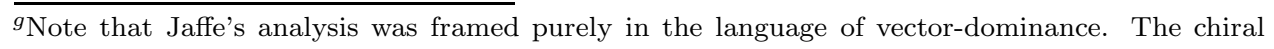
framework which we employ here is taken from Ref. 41. 
where $\epsilon \simeq 0.053 \pm 0.005$ is the mixing angle between the physical $\omega$, $\phi$ and pure $\bar{s} s,(\bar{u} u+\bar{d} d) / \sqrt{2}$ states and is determined from the decay $\phi \rightarrow \pi \gamma$ q. 50 , while $\theta_{0}=\tan ^{-1} 1 / \sqrt{2}$ is the "magic" angle of octet-singlet mixing that would yield such flavor-pure states. In order to obtain the strange residues for the higher mass vector state, Jaffe employed assumptions on the asymptotic dependence of the form factors - that $F_{1}$ vanish as $1 / q^{2}$ and $F_{2}$ as $1 / q^{4}$. In this way he obtained values

$$
\kappa^{s} \simeq-0.3, \quad r_{s}^{2} \simeq 0.2 \mathrm{fm}^{2}
$$

While reasonable and seemingly somewhat model-independent, the difference in sign between the strangeness charge radius obtained in this analysis and that found in those described below should serve as a red flag. In addition, it should be emphasized that the values obtained in the Jaffe analysis are actually quite sensitive to the mixing angle $\epsilon$ and to the assumed representation of the form factors in terms of three poles, two of which are identified in terms of the physical $\omega$ and $\phi$ states. It is particularly sensitive to identification of the second resonance with the $\phi$ because of its very large strangeness content and its relatively large OZI-violating coupling to the nucleon. In fact, the asymptotic dependence of the form factors which results from quark counting arguments is even stronger than assumed in this analysis and would require inclusion of additional dynamical structure. One should also be concerned about the representation of the high energy continuum structure in terms of a simple zero-width pole, since the distribution of strength between strange and non-strange currents is presumably an energy-dependent quantity. One might hope, nevertheless, that low energy static properties should be relatively insensitive to the couplings to high mass states so that perhaps Eq. 72 represents reasonable lowest order estimates. In this regard, Forkel has shown that use of QCD asymptotics can reduce the size of the strangeness couplings by a factor of two to three, although the signs remain fixed.51

\subsection{Kaon loop models}

An alternate approach to the problem of strangeness matrix elements has been to abandon the requirement of a consistent chiral expansion and to simply include a (hopefully reliable) kaon loop contribution. Of course, this must be done carefully since one can see from Eq. 62 that a naive estimate of this type includes divergences. Thus some sort of cutoff procedure must be employed. In fact, this is reasonable. Indeed, recently Donoghue and Holstein (DH) have pointed out that the feature that the baryon has an intrinsic size strongly suggests the use of some sort of regularization which de-emphasizes the effects from heavy meson (and therefore short distance) effects, since such features must surely be suppressed by baryon structure.52 They advocated introduction of a dipole regulator

$$
F\left(q^{2}\right)=\left(\frac{\Lambda^{2}}{\Lambda^{2}-q^{2}}\right)^{2}
$$

into such chiral loop integrals involving baryons and showed that the results of such 
a procedure retained the underlying chiral structure while maintaining the phenomenological success of lowest order chiral predictions, which are often obscured by large nonanalytic contributions from chiral loops. Of course, consistent power counting is lost in such a scheme but the price may be worth paying. The dipole regulator used by DH is similar to use of a form factor and therefore in many ways justifies the simple form-factor modified kaon loop approaches which have appeared in the literature. One complication which must be faced when using a form factor or regulator is the requirement of gauge invariance, which can easily be lost if one is not careful. In order to retain the proper Ward-Takahashi identities it is necessary to include some sort of contact or seagull contribution, whose form is ad hoc. For example, in the case of pseudovector meson-nucleon coupling

$$
F\left(q^{2}\right) q^{\lambda} \phi \bar{u} \gamma_{\lambda} \gamma_{5} u
$$

one can insert a contact term such as 535

$$
\begin{aligned}
& \quad\left\{i A_{\mu}\left(P^{\mu}+2 k^{\mu}\right)\left(\frac{F\left((P-q)^{2}\right)-F\left(q^{2}\right)}{(P+q)^{2}-q^{2}}\right) q^{\lambda}[\hat{Q}, \phi]+i A^{\lambda} F\left((P+q)^{2}\right)[\hat{Q}, \phi]\right\} \\
& \bar{u} \gamma_{\lambda} \gamma_{5} u
\end{aligned}
$$

whose form is, of necessity, model-dependent. 0 Another problem is the size of the cutoff or regulator term, $\Lambda$. In the calculations of DH results were relatively insensitive to the precise size of such a term as long as it was in the range $300 \mathrm{MeV}$ $\leq \Lambda \leq 600 \mathrm{MeV}$, but this is an unavoidable uncertainty since it represents short distance effects which are omitted from the calculation. A precise evaluation of such effects would include additional physics and would be independent of the regulator mass and to the extent that there is a strong dependence on $\Lambda$ such an approximate calculation should be judged to be uncertain. (Thus it is worrisome that there is significant cutoff dependence in the loop analysis performed, e.g., in Ref. 41.)

In any case, various such calculations have been performed. A common feature of any such estimate is that the strangeness charge radius is predicted to be negative since the kaon loop, which carries the $\bar{s}$, is further from the center of mass than is the strange baryon, which carries the $s$ quark, and this is borne out in the calculations. Note, however, as mentioned above, that this is in disagreement with Jaffe's estimate and the simple reasoning given here is based on static arguments and neglects recoil effects. One indication of the importance of such corrections can be seen from the feature that if the cutoff mass is allowed to become very large, corresponding to a pointlike kaon, the strangeness charge radius actually becomes positive, although for the $\Lambda \leq 1 \mathrm{GeV}$ values used in the Bonn potential one finds $r_{s}^{2}<0$. It should also be noted that in general the magnitude of the charge radius predicted in such models is found to be much smaller than in the vector dominance approach. There have been a number of such calculations, whose results are qualitatively similar and which differ only in detail. One such calculation is that of Ramsey-Musolf and Burkardt 5

\footnotetext{
${ }^{h}$ The Ward-Takahashi identity constrains only the longitudinal component of the vertex.
} 
who employed a simple $K \Lambda$ loop calculation using the phenomenological mesonbaryon form factors used by practitioners of the Bonn-Jülich potential 55 (Effects from $K \Sigma$ and $\eta N$ loops were assumed to be much smaller, in accord with $\mathrm{SU}(3)$ symmetry arguments, and were ignored.) Seagull terms, as described in Eq. 75 were included in order to maintain the Ward-Takahashi identities. These authors find results consistent with Jaffe, i.e. a moderate negative value, for the strangeness moment but a smaller and negative value for the strangeness charge radius, as shown in Table 2 .

An alternative approach was taken by Pollock, Koepf, and Henley who employed a cloudy bag model (CBM) to estimate the strangeness moments.56 The simple MIT bag model deals with the confinement issue by incorporating a phenomenologically determined QCD vacuum energy into a theory of noninteracting quarks. 07 Bubbles ("bags" of perturbative vacuum containing such quarks are then allowed to exist and are stabilized against collapse to the $\mathrm{QCD}$ vacuum phase by the pressure exerted via the Heisenberg energy of such quark states. A major failing of the model, however, is that chiral symmetry is not maintained. The CBM is a version of the MIT bag model which incorporates broken chiral symmetry by coupling to mesons at the surface of the bag. In this way an intrinsic cutoff parameter is set by the inverse size of the bag. Results for many static properties of the nucleon has been calculated in such models and results are generally successful. In the case of the vector current strangeness matrix element by Pollock, Koepf, and Henley the results are of the same order as found in the simple kaon loop picture, though the strangeness magnetic moment is somewhat smaller. Since the kaon loop calculation in Ref. 53 utilizes chiral $K-\Lambda$ couplings and a cutoff parameter not dissimilar to the inverse bag radius used in Ref. 56 it is perhaps not surprising that the results are similar.

Cohen, Forkel, and Nielson proposed an approach which unites the phenomenologically successful vector dominance ideas suggested by Jaffe with the intuitively appealing meson cloud picture utilized by Ramsey-Musolf and Burkhardt. Emphasizing that simply adding the results of previous calculations raises important questions involving double counting they considered a "hybrid" model wherein "intrinsic" NN strange and isoscalar matrix elements generated by kaon loops are "renormalized" via use of the current field identity together with phenomenological mixing parameters to yield predictions for corresponding experimental quantities 58 Their results are shown in Table 2 and are similar to the results obtained via kaon loop arguments. Now in fact the agreement in the case of the strangeness magnetic moment is required by the feature that this quantity is obtained at $q^{2}=0$ and consequently receives no contribution from vector meson mixing - it is given purely in terms of the "intrinsic" kaon loop diagrams. The strangeness charge radius on the other hand receives contributions both from the intrinsic component and from the vector meson mixing. The latter considerably enhances the very small negative value found in the kaon loop calculation by a factor of two to three which makes the size much more reasonable from an experimental point of view. As in the Jaffe analysis, however, this result is critically dependent on the size of the parameter $\epsilon$. 


\subsection{Skyrme model}

One of the first pictures used in order to estimate strangeness effects was the Skyrme model. $\mathrm{E}$ In the standard $\mathrm{SU}(2)$ scheme the lowest order chiral Lagrangian, Eq. 41 is augmented by a phenomenological four-derivative term

$$
\Delta \mathcal{L}_{4} \sim \frac{1}{32 e^{2}} \operatorname{tr}\left[\partial_{\mu} U U^{\dagger}, \partial_{\nu} U^{\dagger}\right]^{2}
$$

which stabilizes a classical solition solution

$$
U_{0}=\exp [i F(r) \hat{x} \cdot \vec{\tau}]
$$

against collapse. Allowing time-dependent quantum corrections around this solution via $U=A^{-1}(t) U A(t)$ the Lagrangian reduces to

$$
\mathcal{L}=-M+\lambda \operatorname{tr}\left[\partial_{0} A(t) \partial_{0} A^{-1}(t)\right]
$$

where, in terms of the dimensionless variable $\tilde{r}=2 e F_{\pi} r$

$$
\begin{aligned}
M & \left.=\frac{8 \pi F_{\pi}}{3} \int_{0}^{\infty} \tilde{r}^{2} d \tilde{r}\left\{\frac{1}{8}\left(F^{\prime}\right)^{2}+\frac{2 \sin F}{\tilde{r}^{2}}\right]+\frac{\sin ^{2} F}{2 \tilde{r}^{2}}\left[\frac{\sin ^{2} F}{\tilde{r}^{2}}+2\left(F^{\prime}\right)^{2}\right]\right\} \\
\lambda & =\frac{\pi}{3 e^{3} F_{\pi}} \int_{0}^{\infty} \tilde{r}^{2} d \tilde{r}\left[1+4\left(\left(F^{\prime}\right)^{2}+\frac{\sin ^{2} F}{\tilde{r}^{2}}\right)\right]
\end{aligned}
$$

This system may be solved using variational methods and what results is a remarkably successful picture of nucleon structure. Extension of the Skyrme approach to $\mathrm{SU}(3)$ in order to include strangeness effects is not straightforward and introduces additional model dependence 60 The problem was addressed in in Ref. 61 by appending terms involving nonmininal derivative couplings into the Lagrangian in order to account for flavor symmetry breaking. After the usual quantization in the restricted space of collective and radial excitations, the Hamiltonian is diagonalized while treating the symmetry breaking terms exactly. The results are shown in Table 2 and reveal moderate negative values for both the strangeness charge radius and magnetic moment.

However, there is good reason to be concerned about the accuracy of such predictions. For one thing the extension to $\mathrm{SU}(3)$ involves a number of ambiguities and the choice made by Park et al. in Ref. 61 is in that sense arbitrary. Indeed the Skyrme model is generally acknowledged to be justified in the large- $N_{c}$ limit, so that making reliable predictions concerning subtle quark sea effects in the real $N_{c}=3$ world is a stretch. Another indication of the approximate nature of any Skyrme result is the fact that, as shown by Gasser and Leutwyler, the full four derivative chiral Lagrangian consists of ten such terms and there are even more contributions at six and higher derivatives which must be included in order to fully represent the effective Lagrangian of QCD. Thus any results arising from the use of the simple four-derivative stabilizing term Eq. 76 can be approximate at best. Finally, since 
the Skyrme practitioners evaluate the strangeness current as the difference between the baryon number and hypercharge currents

$$
J_{\mu}^{s}=J_{\mu}^{B}-J_{\mu}^{Y}
$$

any predictions for strangeness matrix elements are obtained by taking the (small) difference of two sizable and themselves uncertain quantities, lending considerable doubt as to their reliability. One indication of this uncertainty is that in a version of the model wherein vector mesons are included, results become smaller by about a factor of two and the sign of the strangeness charge radius changes.62

\subsection{Constituent quark approach}

Still another approach to the problem is to attempt to take the internal quark structure of the nucleon into account by representing it in terms of the usual complement of three constituent $(U, D)$ quarks, whose substructure consists partly of $\bar{s} s$ pairs. This is the calculational manifestation of the suggestions of Kaplan and Manohar. 10 The question is how to take this quark sea structure into account. A simple approach taken by Ramsey-Musolf and Ito is to assume that the constitutent quarks are themselves coupled to mesons via a chiral quark model. 41 In this picture the strangeness content arises from the feature that the $U, D$ quarks can fluctuate into a kaon plus constituent $S$-quark. The axial coupling of the constituent quarks to mesons is determined by a constant $\lambda_{A}$ which is fit by demanding agreement of the chiral quark model calculation with the experimental value of the axial coupling in neutron beta decay $-g_{A} \simeq 1.27$. This procedure too is fraught with model-dependent assumptions, however. Indeed, since such loop calculations are themselves divergent, new counterterms

$$
\Delta \mathcal{L}=\frac{b_{q}^{a}}{2 \Lambda_{\chi}} \bar{\psi} \sigma_{\mu \nu} \hat{Q} \psi F^{\mu \nu}-\frac{c_{q}^{a}}{\Lambda_{\chi}^{2}} \bar{\psi} \gamma_{\mu} \hat{Q} \psi \partial_{\nu} F^{\mu \nu},
$$

corresponding to a magnetic moment and charge radius for the constituent quark, must be determined in terms of experimental quantities. Since the singlet channel quantities $-a_{q}^{0}, b_{q}^{0}$ - are unknown we have no predictive power and are forced to assume something. Using arguments similar to those of DH, Ramsey-Musolf and Ito, for example, inserted a cutoff $\Lambda_{\chi}$ into the loop integrations in order to avoid infinities and assumed the validity of the simple one kaon loop approximation, obtaining the results shown in Table 2. One unfortunate feature of this model, however, as noted by the authors, is that in addition to the usual model-dependence one also has to be concerned about double counting issues. Specifically, it is not clear whether $Q \bar{Q}$ bound states, which must be present in such a model, should be included in or separate from the Goldstone bosons which are included as part of the chiral couplings.

Another chiral quark model calculation, though somewhat more elaborate since it includes both kaon as well as $K^{*}$ and $K^{*}-K$ loops, has been done by the Helsinki group 63 Inclusion of the vector loops somewhat stabilizes the calculation 
against senstivity to the size of the regulator provided that a cutoff of order the chiral symmetry breaking scale is chosen. The authors find a small negative $(\sim-$ 0.05 ) value for the strange magnetic moment, with a substantial cancellation arising between the kaon and $K^{*}$ loop contributions. In this work, besides the remaining cutoff dependence there is also an uncertainty introduced due to the loop diagram involving the $K-K^{*}-\gamma$ vertex.

An alternative approach is to include strangeness in the constituent quark structure via use of Nambu-Jona-Lasinio (NJL) model methods. 4 The NJL model is an effective field theory involving relativistic fermions interacting through four-point vertices subject to the requirements of chiral symmetry. In order to introduce a strangeness component into the valence constituent quarks one requires some sort of flavor mixing interaction. In the work of Refs. 65 and 66 this was accomplished within the mean field (Hartree-Fock) approximation to the NJL model, wherein such terms arise from six-quark interactions involved in the determinant. Such terms are necessary in order to provide the anomalous breaking of $U_{A}(1)$ symmetry implied by 't Hooft's instanton method 67 and involve, in general, constants which must be fit empirically in terms of known singlet-octet and $\rho-\omega$ mixing. There exists considerable model dependence in this process but the $\bar{s} s$ component which is allowed in the $U, D$ quarks is generally small. A specific estimate of the strangeness charge radius by Forkel et al. yields a small positive value of $0.017 \mathrm{fm}^{2} .54$ A related alternative approach is to bosonize the NJL model, yielding an effective Lagrangian containing a topological soliton, which may then be solved via Skyrme methods 68 In this case also there exists considerable model dependence as can be seen from the range of numbers which are allowed by reasonable assumptions ( $c f$. Table 2).

\subsection{Dispersion relations}

Another approach to the subject of strangeness content is through dispersion relations.69 In many ways this technique is like that of effective field theory, since in both cases one takes a method based on general principles - chiral symmetry in the case of chiral perburbation theory and causality and analyticity in the case of dispersion relations in order to interrelate experimental quantities, although an important difference is that the dispersion relations do not involve a systematically controlled approximation. In the case of the strangeness content, for example, one has the relations

$$
\begin{aligned}
r_{s}^{2} & =\frac{6}{\pi} \int_{t_{0}}^{\infty} d t \frac{\operatorname{Im} F_{1}^{s}(t)}{t^{2}} \\
\kappa^{s} & =\frac{1}{\pi} \int_{t_{0}}^{\infty} d t \frac{\operatorname{Im} F_{2}(t)}{t}
\end{aligned}
$$

Here the possible intermediate states which contribute to the spectral function $\operatorname{Im} F_{1}^{s}(t)$ include $3 \pi, K \bar{K}, N \bar{N}$, etc. It is suggestive to focus on the $K \bar{K}$ in particular since this is the lightest state which has no OZI rule suppression. An important complication is the feature that the required spectral density utilizes contributions 
from $N \bar{N} \rightarrow K \bar{K} \rightarrow V_{\mu}$ in the region $4 m_{K}^{2}, t, \infty$, but direct data exist only when $t>4 M-N^{2}$. The solution to this problem is to invoke crossing symmetry and backward dispersion relations in order to produce the needed helicity amplitudes for $N \bar{N} \rightarrow K \bar{K}$ in terms of measured $K N$ scattering data. This program was carried out by Ramsey-Musolf and Hammer 0 who expressed the required spectral densities via

$$
\begin{aligned}
& \operatorname{Im} F_{1}^{s}(t)=\left(\frac{M_{N} \sqrt{\frac{t}{4}-m_{K}^{2}}}{t-4 M_{N}^{2}}\right)\left[\sqrt{\frac{1}{2}} \frac{E}{M_{N}} b_{1}^{1 / 2,-1 / 2}-b_{1}^{1 / 2,1 / 2}\right] F_{K}^{s}(t)^{*} \\
& \operatorname{Im} F_{2}^{s}(t)=-\left(\frac{M_{N} \sqrt{\frac{t}{4}-m_{K}^{2}}}{t-4 M_{N}^{2}}\right)\left[\sqrt{\frac{1}{2}} \frac{M_{N}}{E} b_{1}^{1 / 2,-1 / 2}-b_{1}^{1 / 2,1 / 2}\right] F_{K}^{s}(t)^{*}
\end{aligned}
$$

where $b^{1 / 2, \pm 1 / 2}$ are the helicity amplitudes for the process $K \bar{K} \rightarrow N \bar{N}$ obtained from analytic continuation of $K N$ scattering data, while $F_{K}^{s}(t)$ is the kaon strangeness form factor, which is defined through the matrix element

$$
<0\left|\bar{s} \gamma_{\mu} s\right| K\left(p_{1}\right) \bar{K}\left(p_{2}\right)>\equiv F_{K}^{s}(t)\left(p_{1}-p_{2}\right)_{\mu}
$$

for which no data exists. Thus one must of necessity make some model dependent assumptions in order to perform the requisite dispersive integration. Ramsey-Musolf and Hammer addressed this problem by looking at the related isoscalar electromagnetic form factor where one has data on the vector current $K \bar{K}$ coupling from electron scattering. It is known in this case that the isoscalar magnetic from factor in particular can be well described in terms of opposite sign contributions from $\omega$ and $\phi$ states plus small corrections from higher mass states. (The same is true, but to a lesser extent, for the $q^{2}$ dependence of the charge form factor.) The authors noted that the residues in such a vector-meson pole model fit to the isoscalar electromagnetic form factors could be carried over to the desired strangeness problem using the Jaffe rotation, Eq. 71. Since

$$
\frac{a_{\omega}^{s}}{a_{\omega}^{I=0}} \sim-0.2 ; \quad \frac{a_{\phi}^{s}}{a_{\phi}^{I=0}} \simeq-3
$$

we see that the $\omega$, which is associated with the $3 \pi$ intermediate state, can essentially be neglected, while the $\phi$ plays a dominant role. In this way these authors argued convincingly that the low energy component of the dispersion integral is fairly reliably estimated, leading to a small negative value for $\kappa_{s}$ consistent with original Jaffe estimate. In the case of the strangeness radius, there is somewhat more model dependence. There is also some uncertainty with respect to higher mass contributions. With these caveats the results are listed in Table 2. Note that the strange magnetic moment is roughly consistent with the chiral analyses, while the predicted charge radius is fairly large and positive, $r_{s}^{2} \sim 0.42 \mathrm{fm}^{2}$.

\subsection{Summary of theoretical models}




\begin{tabular}{|c|c|c|}
\hline Model & $r_{s}^{2}\left(\mathrm{fm}^{2}\right)$ & $\kappa_{s}$ \\
\hline Vec. Dom 46 & 0.2 & -0.3 \\
\hline$K \Lambda \operatorname{logp} 53$ & -0.007 & -0.35 \\
\hline CBM대 & -0.011 & -0.10 \\
\hline Hybrid58 & -0.025 & -0.3 \\
\hline chiral quark 41 & -0.035 & -0.09 \\
\hline Skyrme61 & $-0.10--0.15$ & $-0.13--0.57$ \\
\hline Skyrme+VPom. & +0.05 & -0.05 \\
\hline NJL-sol.68 & $-0.25--0.15$ & $-0.05-+0.25$ \\
\hline Dis. Rel.69 & 0.42 & -0.28 \\
\hline
\end{tabular}

Table 2: Calculated values for the strangeness moment and charge radius in various models.

We have seen above that a variety of theoretical approaches have been applied to the problem of calculating matrix elements of $\bar{s} \mathcal{O} s$ operators. This survey is not meant to be exhaustive - indeed there are additional calculational approaches which we have not examined. However, it should be clear from our discussion that any such evaluation must of necessity involve significant model dependence. Indeed one indication of this fact is that the vector dominance analysis of Jaffe, predicts a positive strangeness radius, while most of the others come down on the negative side. Other warning signs are present, too. In each estimation there exist built-in and inescapable assumptions concerning values for cutoff parameters, unconstrained coupling constants, etc. as well as the validity of the simple one loop approach. Although the dispersive evaluation seems somehow more secure, both in it as well as in the Feynman diagrammatic approaches, the authors strongly emphasize the model dependent features which go into their calculations, so that uncertainties are easily at the $\sim 100 \%$ level. This is simply the state of such hadronic calculations at this time. In spite of all the differences and warning signs, however, most of the calculations appear to lead to similar conclusions - a moderate negative strangeness moment, $\kappa^{s} \sim-0.3$ and a small negative strangeness radius, $r_{s}^{2} \sim-0.010 \mathrm{fm}^{2}$.

Lest we become too confident, however, it should be noted that the negative signs for both the strangeness radius and magnetic moment favored by most of the models is opposite in sign to the central numbers measured by SAMPLE and HAPPEX (see Section 6). An additional warning sign comes from the realization that the basis of the above loop calculations is that

i) despite large coupling, rescattering (multi-loop) effects are suppressed.

ii) the lightest hyperon-strange meson intermediate states generate the dominant contribution to the strangeness matrix elements;

Both assertions have been challenged by recent calculations. Questions about the validity of the former are raised by the dispersive evaluation of Ramsey-Musolf, 
Hammer, and Drechsel where rescattering effects are found to be important even at relatively low energies and in order to build up resonance strength in the $\phi$ region. In the case of the latter, a recent calculation by Geiger and Isgur, demonstrated in a simple model calculation that $\bar{s} s$ pair effects result from delicate cancellations between much larger contributions from a significant number of virtual meson-baryon intermediate states rather than being dominated by only the lowest-lying, as assumed in most of the above analvses 71 This worry is consistent with, and amplified by, a calculation by Barz et al., 2 which argues that $K^{*}$-loop effects, whether calculated via dispersive or Feynman diagram methods, are certainly comparable to or in some cases even larger than the corresponding kaon loop effects. The problem may be that present calculations, which rely generally on the assumption of dominance by a few light intermediate states and of neglecting rescattering, are insufficient. If this is borne out by future work, the task of theoretically estimating such quantities and of making contact between experimental numbers and theoretical input for observables involving non-valence sea effects will indeed become even more daunting than it is already. This, of course, is simply an indication of the difficulties of dealing with subtle issues of hadronic physics. Its solution awaits reliable unquenched lattice calculations and/or improved hadronic models/methods and remains one of the important challenges for study of the strong interaction as we enter the twenty-first century.

\section{Experimental Methods}

Since, as described above, there is no convincing way to calculate the strange quark contributions, it must be the task of experiment to provide the information. The strange quark matrix elements described in Section 3 are determined from comparison of the electromagnetic and weak currents as discussed in Section 2.1. The neutral weak currents are measured in parity-violating asymmetry measurements as discussed in Section 2.3. The experimental requirements imposed by the small asymmetries in these measurements center on three areas: statistical precision, systematic accuracy and kinematic selection. The present experiments take advantage of the pioneering parity-violation measurements at SLAd74, MIT-Bates 75 and Mainz 76 There are a number of different realizations in the present experimental program which will be summarized briefly below.

Because the asymmetries range from $\sim 10^{-5}-10^{-4}$ for the $Q^{2}$ range of current experiments $\left(0.1<Q^{2}<1 \mathrm{GeV}^{2}\right)$, there is a strong requirement for high counting rates. All experiments use a liquid hydrogen or deuterium target and an intense electron beam. With target lengths of $10-40 \mathrm{~cm}$ and beam currents of $40-100 \mu \mathrm{A}$, the typical luminosity in these experiments is $\mathcal{L} \geq 10^{38} \mathrm{~cm}^{-2} \mathrm{~s}^{-1}$. In principle, the largest detector solid angle possible is also desirable. This may be done directly as in the SAMPLE experiment, where the acceptance at backward scattering angles

\footnotetext{
${ }^{i}$ This may not be as problematic as it originally seemed since recent work by Forkel, Navarra, and Nielsen using the softer $K^{*}$ form factors favore by recent Bonn-Jülich potential practitioners has substantially weakened these $K^{*}$-loop effects. 3
} 
is $\Delta \Omega \sim 1.5 \mathrm{sr}$, or a large fraction of the azimuthal acceptance may be covered at very small scattering angles as is the case with the HAPPEX experiment where the solid angle acceptance is about $11 \mathrm{msr}$. It should also be noted that the standard figure of merit for these asymmetry measurements

$$
F=P_{\text {beam }}^{2} A_{P V}^{2} \sigma \propto \frac{1}{T_{\text {expt }}}
$$

is essentially independent of momentum transfer (as $A_{P V} \propto Q^{2}$ and $\sigma \propto Q^{-4}$ ) at low momentum transfers where the form factors are of order 1 . The experimental techniques are therefore roughly independent of momentum transfer. In order to measure an asymmetry of $10^{-5}$ with an uncertainty of a few percent, typically 45 days of beam time are required. This time depends critically on the beam polarization of course: the uncertainty achieved is directly proportional to $1 / P_{\text {beam }}$.

With statistical precision approaching $\Delta A_{\text {stat }} \sim 10^{-7}$, the clear challenge is to achieve comparable accuracy in the systematic corrections that must be applied to the measurements. In general, the measured asymmetry must be corrected. To first order

$$
A_{\text {phys }}=A_{\text {meas }}-\sum_{i} \frac{1}{2} \frac{\partial \ln (Y)}{\partial x_{i}} \Delta x_{i}
$$

where $Y$ is the measured yield and the $x_{i}$ are the beam parameters such as position, angle, energy, size, etc. In particular, it is the helicity-correlated differences in these beam parameters, $\Delta x_{i}$, that are necessary to correct the measured asymmetry. In practice, for electron scattering experiments, both the helicity-correlated beam parameter differences and the yield derivatives are measured continuously during the experiment. In order to measure the derivatives two methods may be employed. The natural variation of the beam parameters during a given measurement interval may be used to construct the derivatives to correct the data for that interval, or the beam parameters may be deliberately varied over a wider range and the resulting derivatives utilized.

Of course if the helicity-correlated beam parameter variations vanish, then no corrections would be necessary. Reducing the $\Delta x_{i}$ has required substantial effort, but the results have been encouraging. In fact, generating polarized electron beams that essentially differ only in their helicity is possible because of the exquisite control possible in modern laser beam lines. Polarized electron sources produce beams using the photoelectric effect with circularly polarized laser light. Electrons with positive and negative helicities are generated from either bulk (maximum polarization $50 \%$ ) or strained (maximum practical polarization up to $\sim 90 \%$ at reduced currents) GaAs using right- and left-circularly polarized light, respectively. The light polarization is reversed using an electro-optic $\lambda / 4$ plate whose axes can be rotated rapidly (Pockels cell). Feedback loops are typically used to control the intensity of the right- and left-handed light beams as well as their positions on the photocathode. Great care must be taken, especially with the strained crystals, to reduce the linearly polarized component of the light (which generally changes magnitude 
and/or direction when the circular polarization is reversed) because the strained crystal acts as an optical analyzer. Typical circular polarizations are $99.95 \%$ and the goal for the most effective operation with strained crystals is $99.999 \%$. Using present techniques, helicity-correlated beam position differences at the target are typically reduced to $10 \mathrm{~nm}$ or less and relative helicity-correlated energy differences to $10^{-8}$. It should also be noted that in contrast to proton parity-violation experiments where the beams are non-relativistic, the effects of transverse polarization are small in the electron case, since they are generally reduced by the relativistic $\gamma$ factor.

Because of the large acceptances required to measure these small asymmetries, some attention must be paid to selection of kinematics. The natural variation of the electron scattering cross section is favorable in this case. The general goal is to measure a particular piece of the asymmetry at a particular momentum transfer. At backward electron scattering angles, where the cross section is small, the momentum transfer varies slowly with angle allowing a large solid angle acceptance to be used. At forward scattering angles, the momentum transfer varies rapidly with angle, necessitating a relatively smaller acceptance. However, the forward cross sections are much larger and offset the acceptance. A complete separation of the three neutral weak form factors, $G_{E}^{Z}, G_{M}^{Z}$, and $G_{A}^{Z}$ requires either measurements at three angles or, more practically, measurements at two angles and a measurement of quasi-elastic scattering from deuterium where the combination of asymmetries from the proton and neutron enhance the axial form factor over the strange quark ("unknown") parts of the vector form factors.

\section{Experimental Results}

Following three pioneering parity-violating electron scattering experiments, $74,75,76$ a program has developed to investigate primarily the elastic neutral weak form factors of the nucleon. Two experiments have published results: SAMPLE and HAPPEX. These experiments will continue and be supplemented by several new experiments (including PVA4 and G0) which will be discussed briefly in the following section.

\subsection{SAMPLE experiment}

In the SAMPLE experiment, elastic scattering from the proton 7879 and quasielastic scattering from the deuteron $\$ 0$ are measured at backward angles as shown schematically in Fig. 3. The average momentum transfer of the measurement is 0.1 $\mathrm{GeV}^{2}$, with scattering angles ranging between about $130^{\circ}$ and $170^{\circ}$. The polarized beam from the MIT-Bates accelerator $\left(P_{\text {beam }} \sim 35 \%\right.$ using a bulk GaAs photocathode) with an intensity of $40 \mu \mathrm{A}$ is incident on a $40 \mathrm{~cm}$ liquid hydrogen target. The scattered electrons are measured using a 10 segment air Cerenkov detector (threshold energy of $21 \mathrm{MeV}$ ). Because the instantaneous counting rate in a given detector segment is about $100 \mathrm{MHz}$, the signals are integrated. 


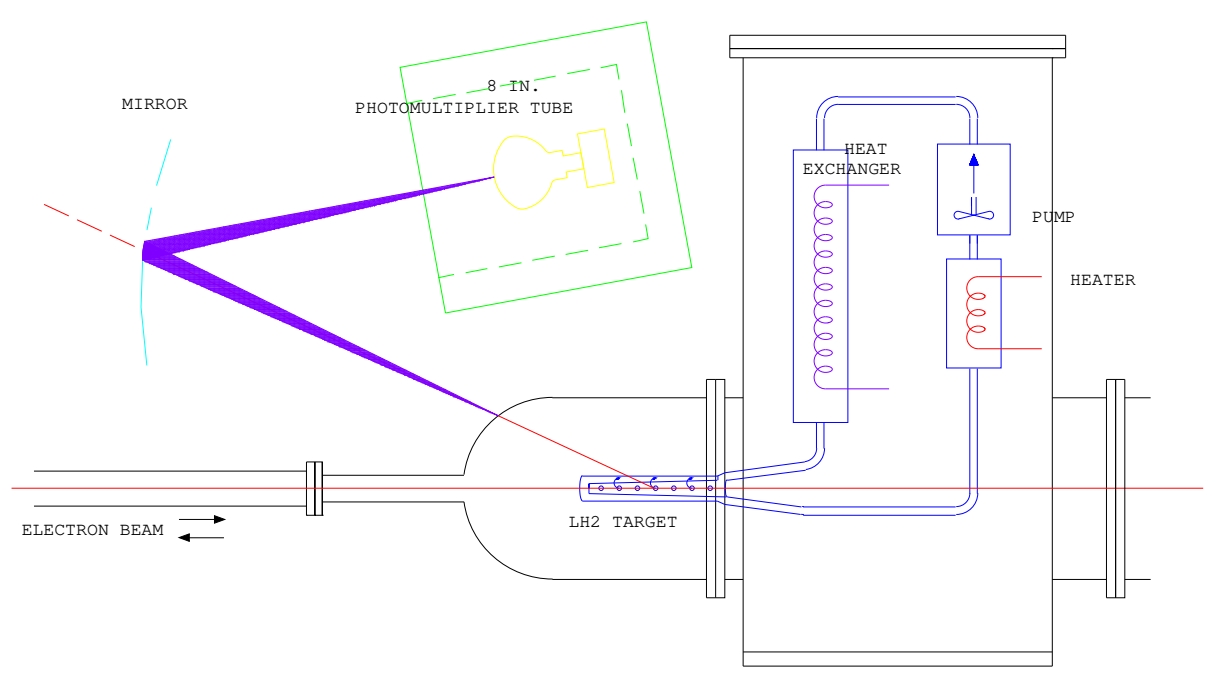

Fig. 3. Schematic representation of the SAMPLE experiment. The detector consists of 10 mirrorphototube pairs to collect the Cerenkov light emitted in air by the scattered electrons.

The measured proton asymmetry is

$$
A_{p}^{e x p}\left(Q^{2}=0.1 \mathrm{GeV}^{2}, \theta_{a v}=146.2^{\circ}\right)=-4.92 \pm 0.61 \pm 0.73 \mathrm{ppm}
$$

where the first uncertainty is statistical and the second systematic. This is to be compared to a "standard" asymmetry of $-7.2 \mathrm{ppm}$ where it is assumed that the strange quark contribution to the vector currents is zero and that the corrections to the axial current are as calculated in Ref. 90. Showing the dependence on the strange quark and axial contributions explicitly, the theoretical asymmetry is

$$
A_{p}^{t h}\left(Q^{2}=0.1 \mathrm{GeV}^{2}, \theta_{a v}=146.2^{\circ}\right)=\left(-5.61+1.37 G_{A}^{e}(T=1)+3.49 G_{M}^{s}\right) \operatorname{ppm}(89)
$$

The systematic uncertainty in this measurement is dominated by contributions other than those associated with false asymmetries. The largest contributions are from background subtractions. Background subtractions for this experiment involve both light from scintillation rather than Cerenkov radiation as well as yield not associated with light (measured with a shutter in front of the each phototube). In particular for the proton measurement, the "shutters closed" and "scintillation" asymmetries contributed essentially all of the $15 \%$ systematic uncertainty. In contrast, the individual contributions from the beam parameter corrections ("false asymmetries") and the beam polarization were about $5 \%$.

A separate measurement was performed with the same apparatus but with a deuterium target. Because of the large energy acceptance of the detector both elastic and quasi-elastic scattering from the deuteron were measured. The elastic 
scattering and threshold electrodisintegration contributions (based on the appropriate fractions of the yield) were estimated to change the measured asymmetry by only about $1 \%$. The measured asymmetry was

$$
A_{d}^{e x p}\left(Q^{2}=0.1 \mathrm{GeV}^{2}, \theta_{a v}=146.2^{\circ}\right)=-6.79 \pm 0.64 \pm 0.51 \mathrm{ppm}
$$

In this case the expected asymmetry is $-8.8 \mathrm{ppm}$ again assuming zero strange quark contribution and the "standard" axial corrections of Ref. 90. Again showing the theoretical asymmetry explicitly in terms of the strange quark and axial contributions

$$
A_{d}^{t h}\left(Q^{2}=0.1 \mathrm{GeV}^{2}, \theta_{a v}=146.2^{\circ}\right)=\left(-7.60+1.93 G_{A}^{e}(T=1)+0.88 G_{M}^{s}\right) \mathrm{ppm}
$$

It can be seen here that whereas the sensitivity of the deuterium measurement to the axial current is similar to that of the proton, the contribution from the strange quarks is significantly smaller (essentially by the ratio $\left.\left(\mu_{p}+\mu_{n}\right) / \mu_{p}\right)$. The systematic uncertainties in this measurement are essentially the same as those for hydrogen with a smaller overall "shutters closed" uncertainty.

The results of these measurements are shown in Figure 4 plotted as a function of the strange quark contribution to the magnetic form factor, $G_{M}^{s}$ and the isovector axial current seen by the electron, $G_{A}^{e}(T=1)$. It can be seen that the result is rather far from the expectation of $G_{M}^{s} \sim-0.3$ and $G_{A}^{e}(T=1)=-0.71 \pm 0.20$. Instead the experimental results for these quantities are

$$
G_{M}^{s}\left(Q^{2}=0.1 \mathrm{GeV}^{2}\right)=0.18 \pm 0.30 \pm 0.30
$$

and

$$
G_{A}^{e}\left(T=1, Q^{2}=0.1 \mathrm{GeV}^{2}\right)=+0.27 \pm 0.46 \pm 0.38
$$

These results may also be cast in terms of the general static electron-quark axial couplings $C_{2 u}$ and $C_{2 d} 24$, which are defined as coefficients in the axial part of the neutral current $e N$ lagrangian

$$
\mathcal{L}_{A}^{Z}=\frac{G_{F}}{2} \sum_{i} C_{2 i} \bar{e} \gamma_{\mu} e \bar{q}_{i} \gamma^{\mu} \gamma^{5} q_{i}
$$

where $i$ runs over the quark flavors. As is the case with $G_{A}^{e}$, the combination of the proton and deuterium measurements is sensitive to the isovector combination $C_{2 u}-C_{2 d}$

$$
\begin{aligned}
C_{2 u}-C_{2 d} & =-\left(1-4 \sin ^{2} \theta_{W}\right) \frac{\left(1+Q^{2} / M_{A}^{2}\right)^{2}}{g_{A}} G_{A}^{e}(T=1) \\
& =+0.015 \pm 0.032 \pm 0.027
\end{aligned}
$$

As would be expected from this equation, the tree level contribution to $C_{2 u}-C_{2 d}$ is $-\left(1-4 \sin ^{2} \theta_{W}\right)=-0.075$. Adding in the corrections of Ref. 90 changes the 


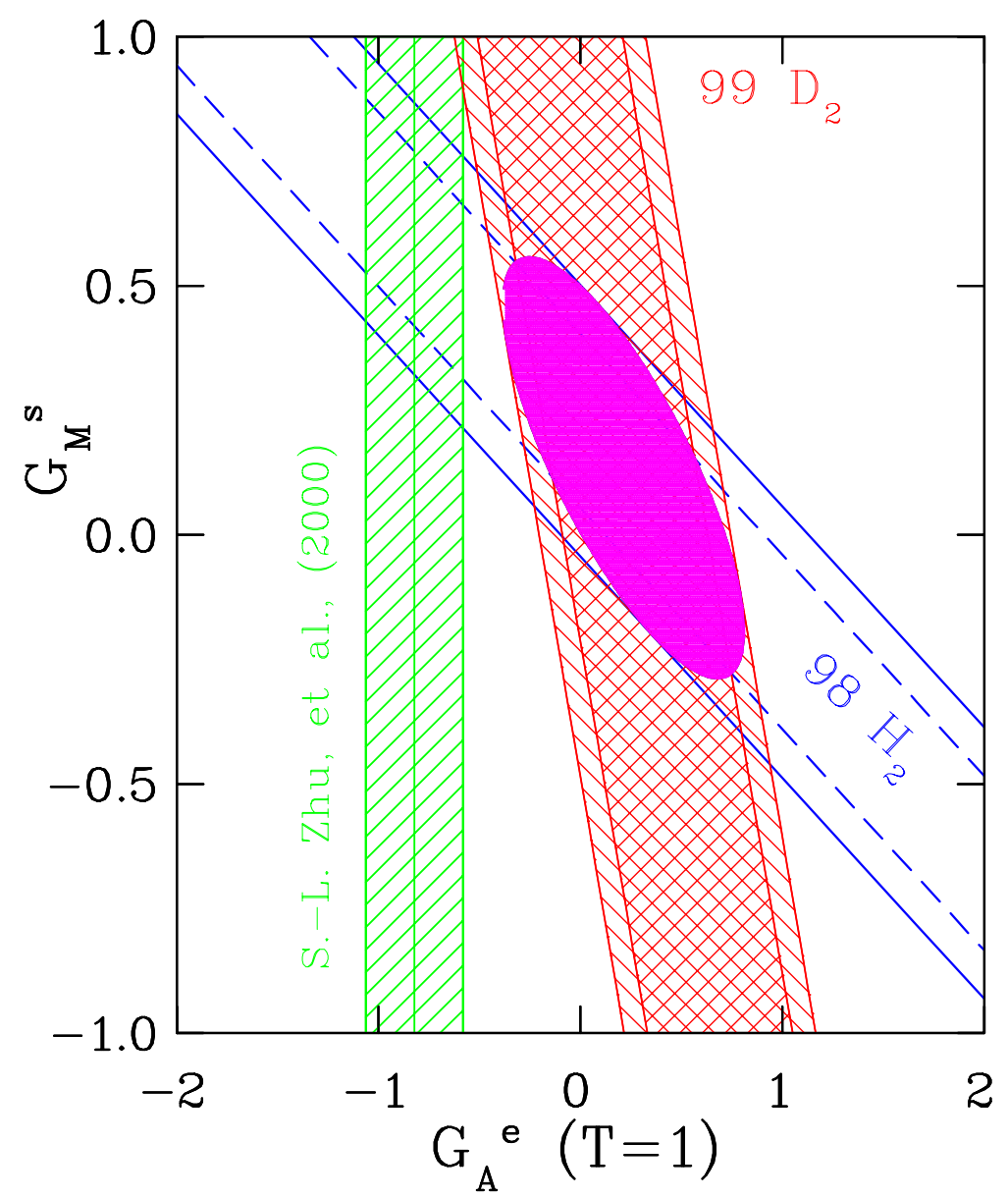

Fig. 4. Pesults from the SAMP华 measurements of parity-violating electron scattering on hydrogen 79 (elastic) and deuterium 80 (quasi-elastic). The contribution of strange quarks to the proton magnetic moment at $Q^{2}=0.1 \mathrm{GeV}^{2}, G_{M}^{s}$ is plotted vs. the effective isovector axial current seen by the electron, $G_{A}^{e}(T=1)$. The vertical band is the theory of Ref. 90 . 
expectation to $-0.058 \pm 0.02$, still about $1.5 \sigma$ from data (cf. the roughly $1.5 \sigma$ deviation of $G_{A}^{e}$ from the calculation in Fig. (4)

A new deuterium measurement is planned 81 at lower momentum transfer to improve the determination of the axial current and check these results. By measuring with an incident energy of $120 \mathrm{MeV}$ rather than $200 \mathrm{MeV}, Q^{2}$ and hence the asymmetry are reduced by a factor of nearly three. However, the cross section will increase significantly relative to the earlier measurement while the background is expected to stay more or less fixed. Because the momentum transfers are so low in both the original and new measurements, it is reasonable to expect the form factors will change in a smooth and predictable manner in this range of momentum transfer. Therefore, in addition to providing an additional measurement of essentially the same physics, some of the critical experimental factors will change thus providing an important cross check.

\subsection{The HAPPEX experiment}

The HAPPEX experiment 82.83 utilized the two spectrometers in Hall A at Jefferson Lab to measure parity-violation in elastic electron scattering at very forward angles. In this case, the relatively small solid angle of each spectrometer, $\Delta \Omega=5.5$ msr, is compensated by the very large $(0.7 \mu \mathrm{b} / \mathrm{sr})$ cross section at forward angles $\left(\theta=12.3^{\circ}\right)$ yielding, with a $15 \mathrm{~cm}$ long liquid hydrogen target, a rate of roughly 1 $\mathrm{MHz}$ in each spectrometer. The scattered electrons were detected by integrating the output of a simple lead-scintillator calorimeter. This calorimeter was shaped to accept only the elastic electrons, which are physically well separated from the inelastic electrons in the focal plane of the spectrometer. The experiment was performed in two stages. The first used a $100 \mu \mathrm{A}$ beam with $39 \%$ polarization produced from a bulk Ga-As crystal. In the second, a strained Ga-As crystal was used, resulting in a beam polarization of about $70 \%$ and a current of $35 \mu \mathrm{A}$, slightly improving the overall figure of merit $\left(\mathrm{P}^{2} \mathrm{I}\right)$.

The measured asymmetry, including the results from both phases of the experiment is

$$
A_{p}\left(Q^{2}=0.477 \mathrm{GeV}^{2}, \theta_{a v}=12.3^{\circ}\right)=-14.60 \pm 0.94 \pm 0.54 \mathrm{ppm}
$$

where again the first uncertainty is statistical and the second systematic. The largest sources of systematic uncertainty are measurement of the beam polarization $(3.2 \%$ of its value) and determination of $Q^{2}$ accruing from uncertainty in measurement of the scattering (spectrometer) angle (contributing a $1.8 \%$ uncertainty to $A_{p}$ ). There is a significant uncertainty in the result owing from uncertainty in the neutron form factors as is shown in Fig. 5 and discussed further below. It should be noted that Fig. 5 suggests that the magnitude of the measured asymmetry is less than that with no strange quarks, in accord with the SAMPLE result.

As is the case for the SAMPLE experiment, the systematic uncertainties due to false asymmetries are very small-negligible, in fact, in this experiment! In principle, the helicity-correlated beam property differences are more pronounced with 


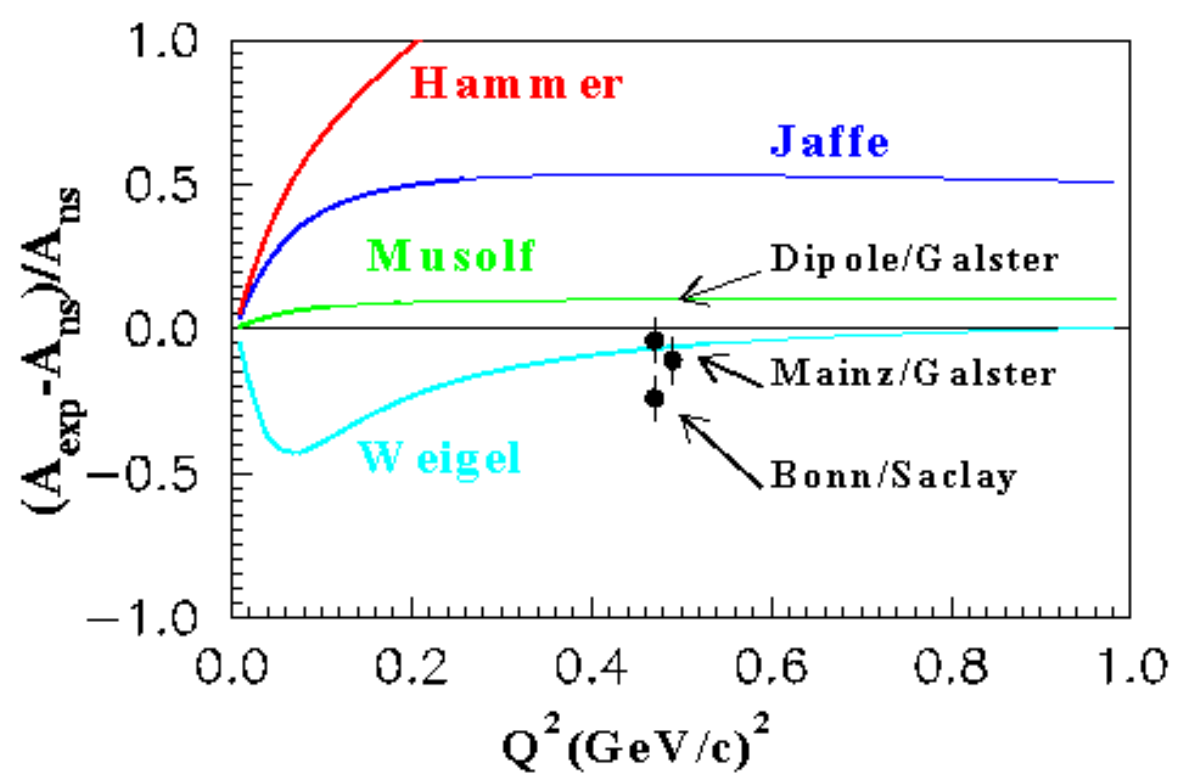

Fig. 5. Results from the HAPPEX measurements of parity-violating electron scattering on hydrogen. The three peints on the plot correspond to the neutron electromagnetic form factors of upper: $G_{E}^{n}$ from Galster 84 and dipole $G_{M}^{n}$; middle $G^{n}$ from Galster and $G_{M}^{n}$ from a Mainz measurement $\$ 5$; and lower: $G_{E}^{n}$ from a Saclay measurement86 and $G_{M}^{n}$ frem a Bonn measurement 87 . The calculations are from Hammer et al.88, Jaffe 46 , Musolf and Itd 41 , and Weigel, et al.89

the strained crystal because it acts as an analyzer of linearly polarized light (always present at some small level in the nominally circularly polarized laser beam-cf. Section 4). However, intensity asymmetries were nulled with a feedback system and position differences were reduced to an acceptable level by tuning the optics of the laser line and by maximizing the "transverse demagnification" of the accelerator. In the HAPPEX experiment, sensitivity to these beam parameter variations was measured by deliberately modulating the beam position and energy. The total correction for beam induced false asymmetries amounted to only $0.02 \pm 0.02 \mathrm{ppm}$ or about $0.1 \%$ of $A_{p}$.

Because the HAPPEX asymmetry was measured at a forward angle, it is in principle sensitive to three unmeasured form factors - $G_{E}^{s}, G_{M}^{s}$ and $G_{A}^{e}$. The axial contribution is relatively small for forward angles (becoming zero at $0^{\circ}$ ) and amounts to $-0.56 \pm 0.23 \mathrm{ppm}$ out of the total of $-14.6 \mathrm{ppm}$, assuming the calculated value 9 for $G_{A}^{e}(T=1)$ rather than that measured in the SAMPLE experiment. The other form factors enter in the combination $G_{E}^{s}+0.392 G_{M}^{s}$ for these kinematics. The value of this combination, normalized to the most accurately measured proton form factor, $G_{M}^{p} / \mu_{p}$, is

$$
\frac{G_{E}^{s}+0.392 G_{M}^{s}}{G_{M}^{p} / \mu_{p}}=0.091 \pm 0.054 \pm 0.039
$$

where the first uncertainty is a combination of the statistical and systematic un- 
certainties in the asymmetry combined in quadrature with the uncertainty in the axial contribution, and the second is due to the uncertainty in the other electromagnetic form factors. 83 The results are particularly sensitive to the neutron magnetic form factor as can be seen in Fig. 5; using the results from a different recent $G_{M}^{n}$ measurement 87 yields

$$
\frac{G_{E}^{s}+0.392 G_{M}^{s}}{G_{M}^{p} / \mu_{p}}=0.146 \pm 0.054 \pm 0.047
$$

Fortunately a number of experiments are planned to reduce the uncertainty in $G_{M}^{n}$.

\subsection{Future experiments}

The HAPPEX group is also approved to make a measurement 91 at a momentum transfer of $Q^{2}=0.1 \mathrm{GeV}^{2}$ utilizing new septum magnets placed in front of the existing spectrometers. These septa will allow measurements at more forward angles (roughly $6^{\circ}$ scattering angle) in order to increase the cross section at low momentum transfers and hence the overall figure of merit. Other recently approved or re-approved parity-violation measurements at JLab include one to determine the neutron radius of the $\mathrm{Pb}$ nucleus 92 and a second to measure the asymmetry in scattering from He at low momentum transfer 93 to measure the proton strangeness radius together with a He measurement at high momentum transfer $94\left(Q^{2}=0.6\right.$ $\mathrm{GeV}^{2}$ ) where early predictions showed a large value of $G_{E}^{s}$. We note that because He is a $0^{+}, T=0$ nucleus, there are neither contributions from $G_{M}$ nor from $G_{A}$, making it particulary advantageous for measurements of $G_{E}^{s}$.

Two new parity-violation experiments are being mounted with dedicated apparatus to address the questions of the weak neutral current in the nucleon. The PVA4 experiment, 95 underway at the MAMI accelerator in Mainz will measure both forward and backward asymmetries using an array of $\mathrm{PbF}_{2}$ calorimeter crystals. The G0 experiment, 96 to be performed at JLab, will also measure at forward and backward angles to separate the contributions of the charge, magnetic and axial terms over the full range of momentum transfers from about $Q^{2}=0.1$ to $Q^{2}=1.0 \mathrm{GeV}^{2}$.

The PVA4 experiment will initially utilize the $855 \mathrm{MeV}$ beam from the MAMI accelerator to measure the parity-violating elastic scattering asymmetry at an angle centered around $35^{\circ}\left(Q^{2}=0.23 \mathrm{GeV}^{2}\right)$. This forward angle asymmetry will yield a measurement of the quantity $G_{E}^{s}+0.21 G_{M}^{s}$; the measurement began in summer 2000. A $20 \mu \mathrm{A}$ beam with $80 \%$ polarization is incident on a $10 \mathrm{~cm} \mathrm{LH}_{2}$ target for the experiment. The detector for the experiment consists of $1022 \mathrm{PbF}_{2}$ calorimeter crystals covering a solid angle of $0.7 \mathrm{sr}$ and arranged in a pointing geometry relative to the target as shown in Figure 6. The first measurements will be made with half the detectors arranged in two diametrically opposed quarters covering half the total azimuthal angle. The fast Cerenkov signal from the $\mathrm{PbF}_{2}$ allows separation of elastic and inelastic electrons in hardware. Using an analog sum of signals from a central detector and its eight nearest neighbors an energy resolution of about $3.5 \%$ has been achieved with an integration gate of $20 \mathrm{~ns}$. This allows effective separation 
of elastic and inelastic electrons - the inelastic yield being about x10 larger than that from elastic scattering. The same apparatus can be reversed relative to the beam to provide corresponding asymmetries over a range of momentum transfers at backward angles (i.e. with a scattering angle of $145^{\circ}$ ).

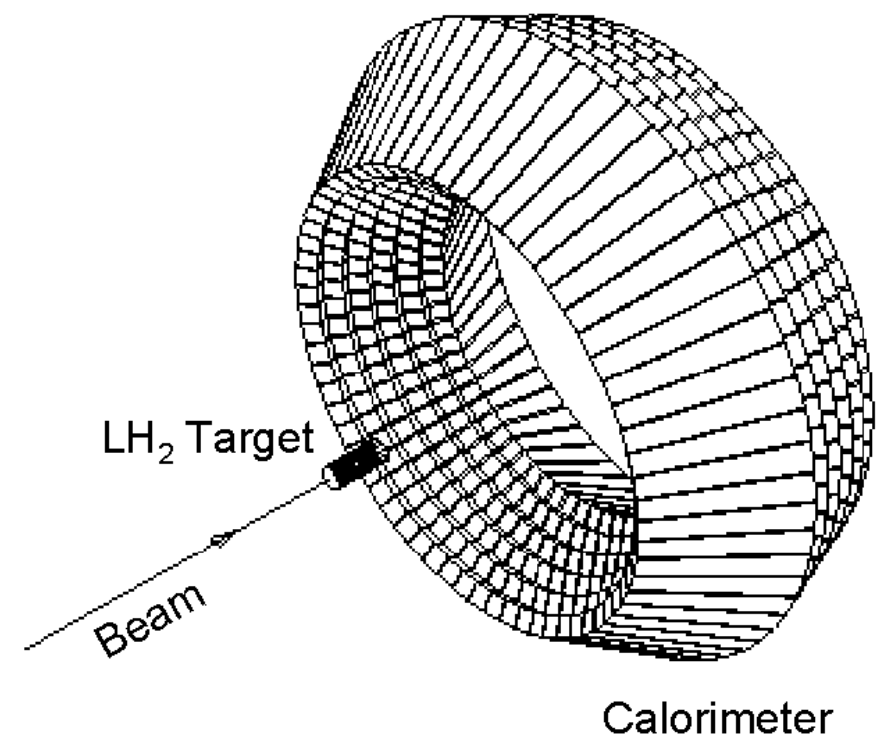

Fig. 6. Schematic of the parity-violating electron scattering experimenta PVA4 being performed at the Mainz Microtron. The calorimeter consists of an array of $1022 \mathrm{PbF}_{2}$ crystals used to count elastically scattered electrons.

The goal of the G0 experiment is to measure forward proton asymmetries and backward asymmetries for both the proton and deuteron in order to provide a complete set of observables from which the charge, magnetic and axial neutral weak currents of the nucleon can be determined. It will utilize a $40 \mu \mathrm{A}, 70 \%$ polarized beam from the JLab accelerator. The experimental apparatus consists of a superconducting toroidal magnet used to focus particles from a $20 \mathrm{~cm}$ liquid hydrogen target to an array of plastic scintillator pairs located outside the magnet cryostat (see Figure 7).

In the first G0 experiment, forward angle asymmetries will be measured by detecting the recoil protons from elastic scattering. With an acceptance of about $0.9 \mathrm{sr}$ (for scattering angles centered at about $70^{\circ}$ ), the spectrometer will measure asymmetries over the range $0.12<Q^{2}<1.0 \mathrm{GeV}^{2}$ with a beam energy of $3 \mathrm{GeV}$. For this measurement elastic protons are identified by time-of-flight (discriminating against inelastic protons and faster $\beta \sim 1$ particles such as $\pi^{+}$) and their $Q^{2}$ is determined by where in the focal surface they are detected. Custom electronics is being used for fast accumulation of t.o.f. spectra with resolutions of $0.25-1$ ns 


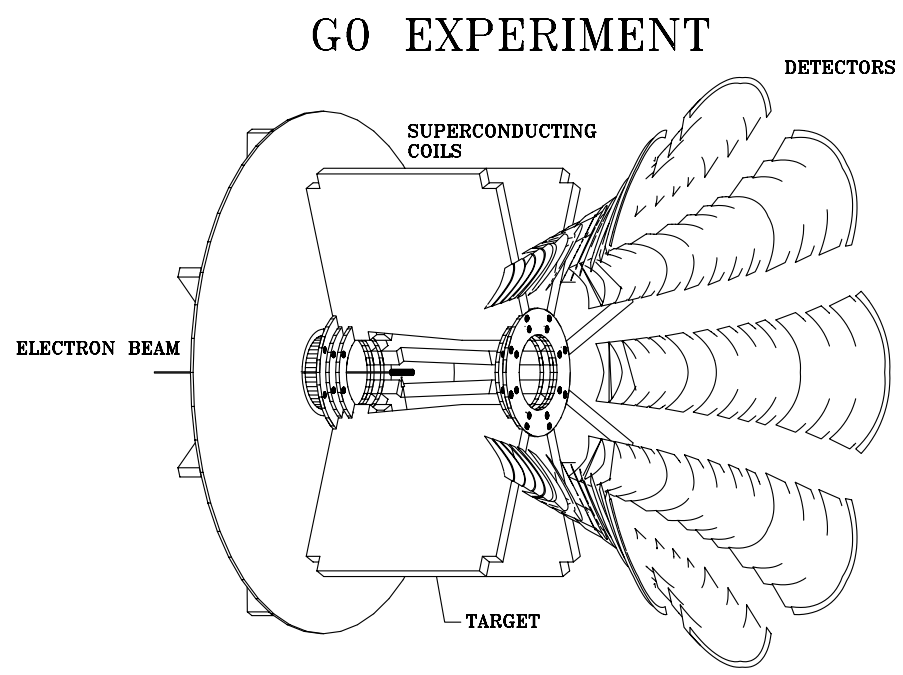

Fig. 7. Schematic of the G0 parity-violating electron scattering experiment to be performed at JLab. A dedicated superconducting toroidal spectrometer will be used to detect recoil protons for forward angle measurements and electrons for back angle measurements.

- the maximum elastic rate in the scintillator pairs is about $1 \mathrm{MHz}$. Background yields and asymmetries are thus measured concurrently and will be used to correct the elastic asymmetries.

Backward angle asymmetries will be measured with the same apparatus, by reversing it relative to the beam direction. In this case elastically scattered electrons will be measured at scattering angles around $110^{\circ}$. A set of smaller scintillators will be installed near the exit window of the cryostat to discriminate elastic and inelastic electrons. In combination with the scintillators in the focal surface this allows a rough measurement of both electron momentum and scattering angle elastic electrons will appear only in certain well defined pairs of detectors. Measurements of quasi-elastic scattering from deuterium at backward angles will require improved particle i.d. to separate electrons and $\pi^{-}$(essentially absent in the hydrogen measurements). In principle, the G0 experiment will therefore also be able to investigate the effective axial current seen by the electron over the full range of momentum transfers of the experiment.

\section{Summary}

The quark and gluon sea of the nucleon is, particularly at low energies, an important, relatively unknown part of its structure. Measurements of strange quark matrix elements of the nucleon represent direct windows on at least part of this 
structure, to the extent that strange and light quarks in the sea have some features in common. Parity-violating electron scattering provides a new, relatively clean determination of the contributions of the three lightest flavors to nucleon vector currents (the ordinary charge and magnetic form factors).

The asymmetries measured in these experiments determine the interference between the weak neutral and electromagnetic nucleon currents. The weak neutral current, in turn, is related to the same matrix elements as the electromagnetic current, but is weighted by the weak charges given by the Standard Model (assuming point-like, spin $1 / 2$ quarks). Thus, by measuring the parity-violating scattering and making one further assumption that the proton and neutron obey charge symmetry, three observables can be used to determine the contributions of the three lightest flavors, in particular those of the strange quarks.

Given the difficulty of including dynamical quarks in lattice QCD calculations, one must presently rely on models to calculate strange quark matrix elements. A number of different, but more or less standard approaches have been made including those associated with chiral perturbation theory, with various hadronic bases (including simple loop diagrams, vector dominance or dispersion relations), with approximations of QCD (Skyrme and Nambu-Jona-Lasinio) as well as with somewhat more microscopic constitutent quark models. There is some consistency among the models, which generally indicate a negative contribution to the magnetic moment and a small strangeness charge radius. However, the agreement of these results with present experiments is not obvious, and in any case the trend of recent investigations is to show that any such predictions are typically very sensitive to some of the assumptions in the calculations. This likely reflects the fact that strange quark matrix elements are rather detailed aspects of a structure whose main features are even difficult to model at this stage. The key question is here is whether any present theoretical scheme can reliably estimate subtle dynamic effects such as the strange matrix elements of the nucleon.

Strange quark vector currents are now being extracted from parity-violation measurements. Even though the asymmetries to be measured are very small - on the scale of parts-per-million - the remarkable precision of polarized electron beams makes such experiments more or less routine. Indeed, in the two completed experiments, the false asymmetries due to helicity-correlated beam changes are essentially negligible compared to other systematic uncertainties. The results from the SAMPLE and HAPPEX experiments suggest small contributions of strange quarks to nucleon matrix elements, perhaps on the scale of $0-10 \%$ of the total. One of the important lessons from the first experiments is that other nucleon form factors are important in extracting this information. The results are, for example, quite sensitive to the neutron magnetic form factor. In addition, the SAMPLE measurement on deuterium indicates that the axial current measured in these experiments has significant differences from that measured in neutrino scattering and that calculations of these effects (related to the nucleon anapole moment) may not be reliable.

Both experiment and theory are at an early stage in this formidable problem. 
The next set of experiments is poised to broaden the range of momentum transfer and to separate the charge and magnetic components of the currents. Particularly because we are at present trying to bootstrap ourselves to a clearer picture of the structure, the basic observables must be measured. Such measurements will provide an important foundation for detailed understanding of forthcoming lattice calculations and their relation to the familiar nucleon models.

\section{Acknowledgement}

It is a pleasure to acknowledge useful input from M.J. Ramsey-Musolf and H. Forkel. This work was supported in part by the National Science Foundation.

[1] See, for example, J. T. Friedman and H. W. Kendall, Ann. Rev. Nucl. Science 22, 203 (1972).

[2] See, for example, R. F. Schwitters and K. Strauch, An.. Rev. Nucl. Science 26, 89 (1976).

[3] For a recent review see, for example, V. Hughes and R. Voss, Ann. Rev. Nucl. Part. Sci. 49, 303 (1999).

[4] R. Frisch and O. Stern, Z. Physik 85, 4 (1933).

[5] R. Hofstadter, Phys. Rev. 35, 48 (1956).

[6] L.A. Ahrens, Phys. Rev. Lett. 35, 785 (1987).

[7] G. T. Garvey, W. C. Lewis and D. H. White, Phys. Rev. C48, 761 (1993), and R. Tayloe, priv. comm.

[8] M. J. Musolf, et al. Phys. Rep. 239, 1 (1994).

[9] R. H. Cahn and F. J. Gilman, Phys. Rev. D5, 1313 (1978).

[10] D. Kaplan and A. Manohar, Phys. Lett. B310, 527 (1988).

[11] T. Adams et al., NuTeV Collaboration, hep-ex/9906038, V. Barone, C. Pascaud, and F. Zomer, hep-ph/0004268

[12] S.J. Brodsky and B.-Q. Ma, Phys. Lett. B381, 317 (1996).

[13] T.P. Cheng, Phys. Rev. D13, 2161 (1976); J. Gasser, H. Leutwyler, and M.E. Sainio, Phys. Lett. B168, 105 (1986).

[14] J. Gasser, Amm. Phys. (NY), 136, 62 (1981); B. Borasoy and U.-G. Meissner, Ann Phys. (NY), 254, 192 (1997).

[15] J. Gasser, H. Leutwyler, and M.E. Sainio, Phys. Lett. B253, 260 (1991).

[16] M. Olsson, Phys. Lett. B482, 50 (2000).

[17] M. Pavan, nucl-th/9912034 (1999).

[18] For a recent update see M.E. Sainio, $\pi N$ Newsletter 13, 144 (1997) and references therein. See also J. Gasser, H. Leutwyler, and M.E. Sainio, Phys. Lett. B253, 252 (1991); P. Büttiker and U.-G. Meissner, Nucl. Phys. A668, 97 (2000).

[19] R. D. McKeown, Phys. Lett. B219, 140 (1989).

[20] D. H. Beck, Phys. Rev. D39, 3248 (1989).

[21] G. A. Miller, Phys. Rev. C57, 1492 (1998).

[22] Ya. B. Zel'dovich, J. Exptl. Theoret. Phys. (U.S.S.R.)33, 1531 (1957).

[23] F. Halzen and Martin. Quarks and Leptons (Wiley, 1984)

[24] C. Caso et al. Eur. Phys. J C3, 1 (1998).

[25] F. Halzen and A. D. Martin, Quarks and Leptons: An Introductory Course in Modern Particle Physics (Wiley, 1984).

[26] M.J. Musolf, Princeton University PhD Thesis (1989); M.J. Musolf and B.R. Holstein, Phys. Rev. D43, 2956 (1991).

[27] E. J. Beise and R. D. McKeown, Comm. Nucl. Part. Phys. 20, 105 (1991).

[28] See, e.g., B.R. Holstein, in Parity Violation in Electron Scattering, ed. E.J. Beise and R.D. McKeown, World Scientific, Singapore (1990). 
[29] P. Geiger and N. Isgur, Phys. Rev. D41, 1595 (1990).

[30] H.-G. Dosch, T. Gousset, and H.J. Pirner, Phys. Rev. D57, 1666 (1998).

[31] J. Gasser and H. Leutwyler, Ann. Phys. (NY) 158, 142 (1984); Nucl. Phys. B250, 465 (1985).

[32] See, e.g. V. Bernard, N. Kaiser, and U.-G. Meissner, Int. J. Mod. Phys. E4, 193 (1995).

[33] J. Goldstone, Nuovo Cim. 19, 154 (1961); J. Goldstone, A. Salam, and S. Weinberg, Phys. Rev. 127, 965 (1962).

[34] M. Gell-Mann, CalTech Rept. CTSL-20 (1961); S. Okubo, Prog. Theo. Phys. 27, 949 (1962).

[35] S. Weinberg, Phys. Rev. Lett. 17, 616 (1966).

[36] See, e.g. B.R. Holstein, Int. J. Mod. Phys. A7, 7873 (1992).

[37] M.L. Goldberger and S.B. Treiman, Phys. Rev. 110, 1478 (1958).

[38] L.L. Foldy and S.A. Wouthuysen, Phys. Rev. 78, 29 (1950).

[39] E. Jenkins and A.V. Manohar, in Effective Theories of the Standard Model, ed. U.-G. Meissner, World Scientific, Singapore (1992).

[40] V. Bernard, N. Kaiser, J. Kambor, and U.-G. Meissner, Nucl. Phys. B388, 315 (1992).

[41] M.J. Ramsey-Mudolf and H. Ito, Phys. Rev. C55, 3066 (1997).

[42] T.R. Hemmert, U.-G. Meissner, and S. Steininger, Phys. Lett. B437, 184 (1998); T.R. Hemmert, B. Kubis, and U.-G. Meissner, Phys. Rev. C60, 045501 (1999).

[43] See, e.g., D.B. Leinweber, Phys. REv. D53, 5115 (1996); S.J. Dong, K.F. Liu, and A.G. Williams, Phys. Rev. D58, 074504 (1998).

[44] D.B. Leinweber, Phys. Rev. D53, 5115 (1996); S.J. Dong and K.F. Liu, Phys. Lett. B328, 130 (1994); S.J. Dong, K.F. Liu, and A.G. Williams, Phys. Rev. D58, 074504 (1998).

[45] G. Ecker et al., Phys. Lett. B223, 425 (1989); Nucl. Phys. B321, 311 (1989).

[46] R.L. Jaffe, Phys. Lett. B229, 275 (1989).

[47] G. Höhler et al., Nucl. Phys. B114, 505 (1976).

[48] P. Mergell, U.-G. Meissner, and D. Drechsel, Nucl. Phys. A596, 367 (1996).

[49] P. Jain et al., Phys. Rev. D37, 3752 (1988).

[50] M. Harada and J. Schechter, Phys. Rev. D54, 3394 (1996).

[51] H. Forkel, Phys. Rev. C56, 510 (1996).

[52] J.F. Donoghue and B.R. Holstein, Phys. Lett. B436, 331 (1998); J.F. Donoghue, B.R. Holstein, and B. Borasoy, Phys. Rev. D59, 036002 (1999).

[53] M.J. Ramsey-Musolf and M. Burkardt, Z. Phys. C61, 433 (1994).

[54] H. Forkel et al., Phys. Rev. C50, 3108 (1994).

[55] See, e.g. R. Machleidt, in Advances in Nuclear Physics, ed. J.W. Negele and E. Vogt, Plenum, New York (1989), Vol. 19.

[56] W. Koepf, S.J. Pollock, and E.M. Henley, Phys. Lett. B288, 11. (1992); W. Koepf and E.M. Henley, Phys. Rev. C49, 2219 (1994).

[57] T. DeGrand, R.L. Jaffe, K. Johnson, and J. Kiskis, Phys. Rev. D12, 2060 (1974).

[58] T. Cohen, H. Forkel, and M. Nielsen, Phys. Lett. B316, 1 (1993).

[59] T.H.R. Skyrme, Proc. Roy. Soc. London, Ser. A260, 127 (1961).

[60] C.G. Callan and I. Klebanov, Nucl. Phys. B262, 362 (1985); M. Chemtob, Nucl. Phys. B256, 600 (1985); H. Yabu and K. Ando, Nucl. Phys. B301, 601 (1988).

[61] N.W. Park, J. Schechter, and H. Weigel, Phys. Rev. D43, 869 (1991).

[62] N.W. Park and H. Weigel, Nucl. Phys. A541, 453 (1992).

[63] L. Hannelius and D.O. Riska, hep-ph/0001325; L. Hannelius, D.O. Riska, and L.Ya. Glozman, Nucl. Phys. A665, 353 (2000).

[64] V. Bernard, R.L. Jaffe, and U.-G. Meissner, Nucl. Phys. B308, 753 (1988).

[65] S. Klimt, M. Lutz, U. Vogl, and W. Weise, Nucl. Phys. A516, 429 (1990).

[66] U. Vogl, M. Lutz, S. Klimt, and W. Weise, Nucl. Phys. A516, 469 (1990). 
[67] G. 't Hooft, Phys. Rev. Lett. 37, 8 (1976); Phys. Rev. D14, 3432 (1976).

[68] H. Wiegel et al., Phys. Lett. B353, 20 (1995).

[69] M.J. Ramsey-Musolf, H.-W. Hammer, and D. Drechsel, Phys. Rev. D55, 2741 (1997).

[70] M.J. Ramsey-Musolf and H.-W. Hammer, Phys. Rev. Lett. 80, 2539 (1998); Phys. Rev. C60, 045205 and 024205 (1999).

[71] P. Geiger and N. Isgur, Phys. Rev. D55, 299 (1997).

[72] L.L. Barz et al., Nucl. Phys. A640, 259 (1998).

[73] H. Forkel, F.S. Navarra, and M. Nielsen, Phys. Rev. C61, 055206 (2000).

[74] C. Y. Prescott, et al. Phys. Lett. B84, 524 (1979).

[75] P. Souder, et al. Phys. Rev. Lett. 65, 694 (1990).

[76] W. Heil, et al. Nucl. Phys. B327, 1 (1989).

[77] C. Sinclair, Jefferson Lab, priv. comm.

[78] B. A. Mueller, et al., Phys. Rev. Lett. 78, 3824 (1997).

[79] D.T. Spayde et al., Phys. Rev. Lett. 84, 1106 (2000).

[80] R. Hasty, et al., Science 290, 2117 (2000).

[81] MIT-Bates experiment 00-04, T. Ito, spokesperson.

[82] K.A. Aniol et al. (HAPPEX Collaboration), Phys. Rev. Lett. 82, 1096 (1999).

[83] K. A. Aniol, et al. (HAPPEX Collaboration), nucl-ex/00060022.

[84] S. Galster, et al. Nucl. Phys. B32, 221 (1971).

[85] H. Anklin, et al. Phys. Lett. B428, 248 (1998).

[86] S. Platchkov, et al. Nucl. Phys. A510, 740 (1990).

[87] E. E. W. Bruins, et al. Phys. Rev. Lett. 75, 21 (1995). \{These data were corrected downward by $25 \%$ of the difference with Ref. 85 by the authors of Ref. 83 based on a private communication with B. Schoch.\}

[88] H.-W. Hammer, et al. Phys. Lett. B367, 323 (1996).

[89] H. Weigel, et al. Phys. Lett. B353, 353 (1995).

[90] See, e.g., S.-L. Zhu et al., Phys. Rev. D62, 033008 (2000); M.J. Musolf and B.R. Holstein, Phys. Rev. D43, 2956 (1991).

[91] Jefferson Lab experiment 99-115, K. Kumar and D. Lhuillier, spokespersons.

[92] Jefferson Lab experiment 00-003, R. Michaels, P. Souder and R. Urciuoli, spokespersons.

[93] Jefferson Lab experiment 00-114, D. Armstrong, spokesperson.

[94] Jefferson Lab experiment 91-004, E. Beise, spokesperson

[95] Mainz experiment PVA4, D. von Harrach, spokesperson; F. Maas, contact person.

[96] Jefferson Lab experiment 00-006, D. Beck, spokesperson. 